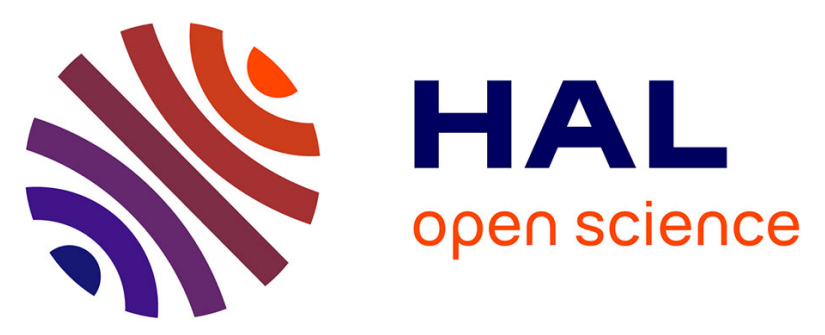

\title{
Characterization of the Drosophila Adult Hematopoietic System Reveals a Rare Cell Population With Differentiation and Proliferation Potential
}

Manon Boulet, Yoan Renaud, François Lapraz, Billel Benmimoun, Laurence Vandel, Lucas Waltzer

\section{To cite this version:}

Manon Boulet, Yoan Renaud, François Lapraz, Billel Benmimoun, Laurence Vandel, et al.. Characterization of the Drosophila Adult Hematopoietic System Reveals a Rare Cell Population With Differentiation and Proliferation Potential. Frontiers in Cell and Developmental Biology, 2021, 9, pp.739357. 10.3389/fcell.2021.739357 . hal-03429562

\section{HAL Id: hal-03429562 https://hal.science/hal-03429562}

Submitted on 15 Nov 2021

HAL is a multi-disciplinary open access archive for the deposit and dissemination of scientific research documents, whether they are published or not. The documents may come from teaching and research institutions in France or abroad, or from public or private research centers.
L'archive ouverte pluridisciplinaire HAL, est destinée au dépôt et à la diffusion de documents scientifiques de niveau recherche, publiés ou non, émanant des établissements d'enseignement et de recherche français ou étrangers, des laboratoires publics ou privés. 


\section{OPEN ACCESS}

Edited by:

Dominic C. Voon,

Kanazawa University, Japan

Reviewed by:

Tomomasa Yokomizo,

Kumamoto University, Japan Jin Li Hua,

Northeast Forestry University, China

*Correspondence:

Lucas Waltzer

lucas.waltzer@uca.fr

${ }^{\dagger}$ Present address:

François Lapraz,

Université Côte d'Azur, Centre

National de la Recherche Scientifique, Institut National de la Sante et de la

Recherche Medicale, Institut de

Biologie Valrose, Nice, France

Billel Benmimoun,

Institut Pasteur, Centre National de la

Recherche Scientifique, UMR 3738,

Paris, France

Specialty section:

This article was submitted to

Cell Growth and Division,

a section of the journa

Frontiers in Cell and Developmental

Biology

Received: 10 July 2021

Accepted: 22 September 2021

Published: 13 October 2021

Citation:

Boulet M, Renaud Y, Lapraz F,

Benmimoun $B$, Vandel $L$ and

Waltzer L (2021) Characterization

of the Drosophila Adult Hematopoietic

System Reveals a Rare Cell

Population With Differentiation

and Proliferation Potential.

Front. Cell Dev. Biol. 9:739357.

doi: 10.3389/fcell.2021.739357

\section{Characterization of the Drosophila} Adult Hematopoietic System Reveals a Rare Cell Population With Differentiation and Proliferation Potential

\author{
Manon Boulet ${ }^{1}$, Yoan Renaud ${ }^{1}$, François Lapraz ${ }^{2 \dagger}$, Billel Benmimoun ${ }^{2 \dagger}$, \\ Laurence Vandel ${ }^{1}$ and Lucas Waltzer ${ }^{1,2 *}$ \\ ${ }^{1}$ Université Clermont Auvergne, Centre National de la Recherche Scientifique, Institut National de la Sante et de la \\ Recherche Medicale, Institut Génétique Reproduction et Développement, Clermont-Ferrand, France, ${ }^{2}$ Centre de Biologie du \\ Développement, Centre de Biologie Intégrative, Université de Toulouse, Centre National de la Recherche Scientifique, \\ Université Paul Sabatier, Toulouse, France
}

While many studies have described Drosophila embryonic and larval blood cells, the hematopoietic system of the imago remains poorly characterized and conflicting data have been published concerning adult hematopoiesis. Using a combination of blood cell markers, we show that the adult hematopoietic system is essentially composed of a few distinct mature blood cell types. In addition, our transcriptomics results indicate that adult and larval blood cells have both common and specific features and it appears that adult hemocytes reactivate many genes expressed in embryonic blood cells. Interestingly, we identify a small set of blood cells that does not express differentiation markers but rather maintains the expression of the progenitor marker domeMeso. Yet, we show that these cells are derived from the posterior signaling center, a specialized population of cells present in the larval lymph gland, rather than from larval blood cell progenitors, and that their maintenance depends on the EBF transcription factor Collier. Furthermore, while these cells are normally quiescent, we find that some of them can differentiate and proliferate in response to bacterial infection. In sum, our results indicate that adult flies harbor a small population of specialized cells with limited hematopoietic potential and further support the idea that no substantial hematopoiesis takes place during adulthood.

Keywords: hematopoiesis, blood cell progenitor, Drosophila, imago, Collier

\section{INTRODUCTION}

Several aspects of blood cell development and functions are conserved between mammals and Drosophila (Hartenstein, 2006; Banerjee et al., 2019). Hence, this insect has been extensively used as a simple genetic model organism to study the fundamental bases underlying hematopoiesis and blood cell functions (Letourneau et al., 2016; Boulet et al., 2018). Yet, while much effort has been devoted to the characterization of Drosophila blood cells at the embryonic and larval stages, the 
adult stage remains less well characterized and conflicting data have been published (see below). Thus, further work is essential to gain a better understanding of the composition and dynamics of the adult hematopoietic system.

As in vertebrates, Drosophila hematopoiesis occurs in successive waves and stems from mesoderm-derived blood cell progenitors (called prohemocytes) (Banerjee et al., 2019). These progenitors can give rise to three main differentiated cell types, collectively called hemocytes and related to vertebrate myeloid cells: plasmatocytes, crystal cells, and lamellocytes (Gold and Bruckner, 2015; Parsons and Foley, 2016). Plasmatocytes are macrophages that form the bulk of the population; they are essentially implicated in tissue remodeling and in the cellular immune response, while crystal cells are implicated in clotting and melanization (an insect-specific immune response). Lamellocytes are normally barely present but their differentiation is massively induced in the larvae in response to pathological situations, such as infestation by parasitoid wasp eggs.

Plasmatocytes and crystal cells are first produced in the embryo after a small group of prohemocytes emerges from the early head mesoderm and differentiates (Tepass et al., 1994; Lebestky et al., 2000; Bataille et al., 2005). These embryo-derived hemocytes compose the larval peripheral blood cells, which are observed either as free circulatory cells in the hemolymph or attached under the cuticle (Holz et al., 2003; Makhijani et al., 2011). Interestingly, differentiated larval plasmatocytes can not only proliferate but also transdifferentiate into crystal cells (Makhijani et al., 2011; Leitao and Sucena, 2015), and it was proposed that the increase in peripheral blood cells during larval development relies on self-renewing plasmatocytes (Gold and Bruckner, 2015). Yet, undifferentiated blood cells were also described among peripheral hemocytes (Sinenko et al., 2010) and recent single-cell sequencing experiments suggest that proliferative plasmatocytes retain a progenitor signature (Cattenoz et al., 2020, 2021; Tattikota et al., 2020). In parallel, a second wave of hematopoiesis takes place during the larval stages in a specialized organ called the lymph gland (Lanot et al., 2001). The lymph gland precursors derive from the embryonic lateral mesoderm and develop in close association with the anterior part of the dorsal vessel (Crozatier et al., 2004; Mandal et al., 2004). In third instar larvae, the lymph gland is composed of three to four pairs of lobes separated by pericardial cells (Lanot et al., 2001; Rodrigues et al., 2021). The posterior lobes are essentially composed of prohemocytes (Rodrigues et al., 2021), while the anterior lobes contain blood cells progenitors, their differentiated progenies (plasmatocytes and crystal cells), and a small cluster of cells that form a niche, called the posterior signaling center (PSC) (Jung et al., 2005; Letourneau et al., 2016). In normal situations, lymph gland blood cells are released into circulation during pupation when the lymph gland disperses (Honti et al., 2010; Grigorian et al., 2011).

By comparison, the adult hematopoietic system of the fly remains poorly characterized (Banerjee et al., 2019). Initial examinations failed to reveal the presence of an hematopoietic organ but showed that hemocytes are scattered throughout the imago, mostly as sessile populations, and accumulate along the heart in the abdomen (Elrod-Erickson et al., 2000;
Lanot et al., 2001), a general feature among insects (Yan and Hillyer, 2020). In addition, it seemed that all the hemocytes were non-dividing phagocytic cells whose number and phagocytic activity decrease with age (Lanot et al., 2001; Mackenzie et al., 2011; Woodcock et al., 2015). Besides, transplantation experiments showed that both embryonic and larval hematopoietic anlagen contribute to the adult blood cells (Holz et al., 2003). Hence, the prevailing view was that the adult hematopoietic system was solely composed of mature plasmatocytes derived from the embryonic and larval stages, and that no hematopoiesis took place during adulthood. Yet, some data suggested a more contrasted situation. First, the analysis of different markers revealed that crystal cells and distinct subpopulations of plasmatocytes are present in the adult (Kurucz et al., 2007; Clark et al., 2011). Second, a seminal study by Ghosh et al. (2015) suggested that hematopoiesis also occurs in the imago. Indeed, the authors proposed that blood cell progenitors capable of differentiation persist in the adult and they observed plasmatocyte proliferation in response to Escherichia coli infection (Ghosh et al., 2015). However, these findings were refuted by a subsequent publication (Sanchez Bosch et al., 2019), in which the authors disproved the claim that the GATA factor Serpent (Srp) is a marker of adult blood cell progenitors and found no evidence for blood cell proliferation or differentiation.

Here, we set out to better characterize the Drosophila adult hematopoietic system. First, we established the gene expression profile of adult hemocytes and compared it to their larval ascendant (larval peripheral hemocytes and lymph gland) to define the common and specific features of these immune cells. Next, we used a panel of hematopoietic markers to assess their expression in the imago and to gain a better appreciation of the adult blood cell landscape diversity and evolution with age. Finally, we focused our analysis on a small population of adult blood cells that does not express hemocyte differentiation markers. We show that they originate from the PSC rather than from larval blood cell progenitors and that they can proliferate and differentiate in response to infection albeit unfrequently. These PSC-derived cells most probably account for the previously reported population of adult blood cell progenitor. Our findings are discussed in view of the current controversies in the field.

\section{MATERIALS AND METHODS}

\section{Fly Strains}

The following strains were used in this study: $w^{1118}$ (BL3605), srpD-GAL4 (Waltzer et al., 2002; Crozatier et al., 2004), srpHemo-H2A-3xmCherry (Gyoergy et al., 2018) (BL78361), Hml - -GAL4 (Sinenko and Mathey-Prevot, 2004) (BL30141 and BL30140), crq-GAL4 (Olofsson and Page, 2005) (BL25041), lz-GAL4 (Lebestky et al., 2000) (BL6313), sn-GAL4 (AvetRochex et al., 2010; Zanet et al., 2012), dot-GAL4 (Kimbrell

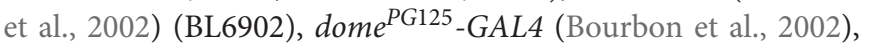
domeMeso-GAL4 (Oyallon et al., 2016), tep4-GAL4 (Avet-Rochex et al., 2010) (DGRC \#105442), Ance $e^{M i M i C}-G F P$ (BL59829), pcol85-GAL4 (Crozatier et al., 2004), col(GMR13A11)-GAL4 (BL49248), Antp-GAL4 (Mandal et al., 2007), Ubx(M3)-GAL4 
(de Navas et al., 2006), Pxn-GAL4 (Stramer et al., 2005), Pxn-RedStinger (Rodrigues et al., 2021), BcF2-GFP (Gajewski et al., 2007), BcF6-mCherry (Tokusumi et al., 2009a), msnF9mCherry, msnF9-GFP (Tokusumi et al., 2009b), Cg-GAL4 (Asha et al., 2003) (BL7011), Cg25C-GFP (Sorrentino et al., 2007), UAS-RNAi col (Baumgardt et al., 2007), $k n^{\text {coll }}$ and $k n^{\text {coll }} P\{$ col5-cDNA $\}$ (Crozatier and Vincent, 1999), UAS-Bax (Gaumer et al., 2000), hop Tum-l (BL8492), UAS-2xEGFP (BL6874), UAS-2xEYFP (BL6660) UAS-RedStinger (BL8546), UAS-mCD8GFP (BL5138), UAS-RNAi srp (VDRC109521), tubP-Gal80 ${ }^{\text {ts }}$ (BL7018), G-TRACE (UAS-RedStinger, UASFLP, Ubi-p63E(FRT.STOP)Stinger) (BL28281), UAS-FUCCI (UAS-GFP.E2f1.1-230, UAS-mRFP1.NLS.CycB.1-266) (BL55110).

Drosophila stocks and crosses were maintained on standard fly medium (75 g/l organic corn flour, $28 \mathrm{~g} / \mathrm{l}$ dry yeast, $40 \mathrm{~g} / \mathrm{l}$ sucrose, $8 \mathrm{~g} / \mathrm{l}$ agar, $10 \mathrm{ml} / \mathrm{l}$ Moldex 20\%). All crosses and collections were performed at $25^{\circ} \mathrm{C}$ with the exception of

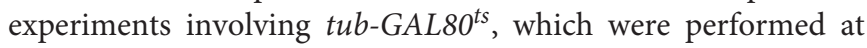
$18^{\circ} \mathrm{C}$ before transferring the progenies to $29^{\circ} \mathrm{C}$ as indicated in the result section.

\section{Adult Blood Cells Preparations}

To bleed adult flies, aged-matched mated individuals were anesthetized, washed in $70 \%$ ethanol and air dried before cutting the last abdominal segment with a clean scalpel. Then a fine glass needle was inserted in the anterior part of the thorax and PBS was perfused under air pressure. Flushed hemocytes were collected in a 12-well plate (Nunc) containing $600 \mu \mathrm{l}$ PBS and a round glass coverslip. In general, six drops of PBS $(\sim 60 \mu \mathrm{l})$ were collected per fly and five flies were bled in a single well. The plates were centrifuged at $1000 \mathrm{rpm}$ for $2 \mathrm{~min}$ before adding $300 \mu \mathrm{l}$ of $16 \%$ formaldehyde for $30 \mathrm{~min}$. For in situ observation of blood cells in adult abdomen, flies were dissected essentially as described in Ghosh et al. (2015). Briefly: flies were anesthetized, stuck on their dorsal side in a paraffin-coated plastic dish and dissected in PBS. The wings and most of the thorax were discarded and the ventral abdomen was incised on both sides with thin scissors before delicately removing the gonads and the gut. The dissected abdomens were fixed in $4 \%$ formaldehyde for $30 \mathrm{~min}$. Samples were then processed as described below for immunofluorescence and/or confocal imaging.

\section{Immunostainings and in situ Hybridizations}

Immunostainings and RNA in situ hybridizations were essentially performed as described in Miller et al. (2017). The following antibodies were used: mouse anti-P1/NimC1 (Kurucz et al., 2007), mouse anti-Col (Krzemien et al., 2007), mouse anti$\beta$ integrin/Myospheroid (CF6.G11, DSHB), mouse anti-Antp (8C11, DSHB), rabbit anti-GFP (Torrey Pines), anti-DIG coupled to alkaline phosphatase (Roche) and Alexa-fluor labeled secondary antibodies (Molecular Probes). Nuclei were stained with DAPI and the actin cytoskeleton with fluorescent labeled phalloidin (Molecular Probes). The slides were mounted in Vectashield (Vector Laboratories) and observed under a confocal microscope (Zeiss LSM800 or Leica SP5). Confocal images are displayed as maximum intensity projection of Z-stacks.

\section{Bacterial and Wasp Infections}

Escherichia coli (DH5 $\alpha$ strain) were grown overnight in LB broth and pelleted at $4000 \mathrm{rpm}$ for $15 \mathrm{~min}$. A fine tungsten needle was dipped in the bacterial pellet and inserted into the dorsal abdomen or under the wing hinge of anesthetized 1week-old females. A heat-sterilized tungsten needle was used to prick the flies that were used as non-infected control. Three groups of 10 flies were used in each experiment. The flies were then reared for $16 \mathrm{~h}$ (FUCCI experiments) to $96 \mathrm{~h}$ (G-TRACE experiments) and dissected or bled as described above. For wasp infestations, late second instar larvae were subjected to parasitism by $\sim 10$ Leptopilina boulardi (strain G486) females for $2 \mathrm{~h}$, as described previously (Benmimoun et al., 2015). Successful infestation was assessed by looking for melanotic nodule $48 \mathrm{~h}$ later. The corresponding larvae were transferred to fresh vial and the surviving adult flies were bled 5 days after emergence.

\section{EdU Incorporation Assays}

For EdU incorporation assays, 2-day-old domeMeso-GAL4, UASEYFP females were grown on $2 \mathrm{ml}$ of fly food medium supplemented with $0.4 \mathrm{mM}$ EdU (5-ethynyl-2'-deoxyuridine) for 5 days before infection with $E$. coli as described above. After $24 \mathrm{~h}$ on medium containing fresh EdU, infected and control flies were bled and EdU incorporation in hemocytes was assessed using Click-IT Plus EdU kit with AlexaFluor 555 dye according to the manufacturer's instructions (Molecular Probes \#C10638).

\section{RNA-Sequencing}

For RNA-seq experiments, wandering third instar larvae or 4- to 5 -day-old adult $w^{1118}$ females were used to prepare independent biological triplicates. Adult blood cells were retrieved by perfusing the flies with PBS as described above and directly collected in a $1.5 \mathrm{ml}$ Eppendorf tube on ice. Peripheral larval blood cells were collected by delicately peeling the dorsal cuticle with forceps and dripping the larva in a $5 \mu \mathrm{l}$ drop of PBS on parafilm. Upon microscopic examination, we found that $>90 \%$ of the cells retrieved with this protocol were hemocytes (PxnRedStinger ${ }^{+}$), with no visible contamination by fat body cells. The anterior lobes of the larval lymph glands were dissected in PBS. Blood cells and lymph glands were transferred to $1.5 \mathrm{ml}$ Eppendorf tubes, pelleted by centrifugation at $1000 \mathrm{rpm}$ for $2 \mathrm{~min}$ and processed for RNA extraction using Arcturus PicoPure RNA kit (ThermoFisher \#KIT0204). For each replicate, $\sim 100$ adult flies and 20 larvae were used. RNA samples were run on Agilent Bioanalyzer to assess sample quality and concentration. Samples were converted to cDNA using Nugen's Ovation RNA-Seq System (Catalog \# 7102-A01). Libraries were generated using Kapa Biosystems library preparation kit (\#KK8201) and multiplexed libraries were sequenced on a 1x75 flow cell on the HiSeq2000 device (Illumina). Reads were filtered and trimmed to remove adapter-derived or low-quality bases using Trimmomatic and checked again with FASTQC. Illumina reads were aligned to Drosophila reference genome (dm6 Ensembl release 70) with Hisat2. Read counts were 
generated for each annotated gene using HTSeq-Count. RPKM (Reads Per Kilobase of exon per Megabase of library size) values were calculated using Cufflinks. Reads normalization, variance estimation and pair-wise differential expression analysis with multiple testing correction was conducted using the $\mathrm{R}$ Bioconductor DESeq2 package. Heatmaps and hierarchical clustering were generated with "pheatmap" R package. Gene ontology enrichment analyses were performed using Genomatix. The RNA-seq data were deposited on GEO under the accession number GSE174107.

\section{Real-Time Quantitative Polymerase Chain Reaction}

For RT-quantitative polymerase chain reaction (qPCR), RNA samples were prepared from adult or larval bleeds and larval lymph glands (dissected as described above) using RNeasy kit (Qiagen) with an additional on-column DNAse treatment with RNase-Free DNase Set (Qiagen). Reverse transcription was performed with SuperScript IV Reverse Transcriptase (ThermoFisher) according to manufacturer's instruction on $100 \mathrm{ng}$ of RNA and using a mix (1:1) of random primers (Invitrogen) and oligo dT (Promega). qPCRs were performed with SsoFast EvaGreen (Biorad) on a LightCycler 480 Instrument II (Roche Life Science). The sequences of the primers used to assess the expression of the different genes are provided in Supplementary Table 1. qPCR data were analyzed with $\Delta \Delta \mathrm{Ct}$ method and gene expressions were normalized to $r p 49$ (synonym: RpL32). All experiments were performed using biological triplicates.

\section{RESULTS}

\section{Adult Blood Cells Exhibit a Distinct Gene Expression Profile as Compared to Larval Blood Cells}

As a first step to characterize Drosophila adult hematopoietic system, we used a perfusion protocol similar to the one used to bleed mosquitoes (Muller et al., 1999) in order to retrieve adult blood cells and define their gene expression program (see the section "Materials and Methods"). We found that 99\% of the cells collected with this technique in 5-day-old flies expressed the pan-hemocyte marker srp as revealed by using the $\operatorname{srpD-GAL4}$ driver (Waltzer et al., 2002) or the srpHemo-H2A::3mCherry reporter (Gyoergy et al., 2018; Figures 1A,C,E), indicating that this approach allows to obtain clean blood cell preparations. Of note, when using young adults (24-48 h after eclosion), the bleeds were contaminated with remnants of the larval fat body (Figure 1B, arrow), which is known to dissociate into large individual adipose cells during metamorphosis (Nelliot et al., 2006) and gets cleared in the first 2 days of adulthood (Aguila et al., 2007). Using aged flies (35-day-old), we found that $\sim 4.6 \%$ of the cells did not express $\operatorname{srp}$ and we recovered fewer hemocytes (Figures 1D-F), which is consistent with previous reports showing that blood cell number decreases with aging (Mackenzie et al., 2011; Woodcock et al., 2015; Sanchez Bosch et al., 2019).

Next, we established the gene expression profile of adult hemocytes collected from 5-day-old flies by RNA sequencing. For comparison, we also established the transcriptome of third instar larval peripheral hemocytes (i.e., embryo-derived hemocytes) and lymph gland hemocytes. All experiments were performed in biological triplicates and at least 14 million mapped reads per sample. We found that 6802 genes were expressed with a RPKM > 1 in all three samples of adult hemocytes (corresponding to $39 \%$ of the genes in Drosophila reference genome dm6) (Supplementary Table 2). Similarly, larval peripheral blood cells and lymph gland hemocytes expressed 6282 and 6220 genes (Supplementary Tables 3, 4), respectively. As shown in Figure $\mathbf{2 A}$, adult hemocytes (AD), lymph gland (LG) and larval peripheral hemocytes $(\mathrm{PH})$ shared the expression of 5351 genes, i.e., between 79 and $86 \%$ of their respective transcriptome. Among the 268 genes annotated in Flybase (FB_2021_02) as being expressed in blood cells (i.e., hemocytes, plasmatocytes, crystal cells, lamellocytes or prohemocytes, Supplementary Table 5), 216 were retrieved in our adult hemocyte data set (2.1-fold over-enrichment, $p$-value $<5.5 \mathrm{E}-45), 197$ in the lymph gland (2.1-fold overenrichment, $p$-value $<3.5 \mathrm{E}-37$ ), and 198 in peripheral hemocytes (2.0-fold over-enrichment, $p$-value $<3.4 \mathrm{E}-37)$, with 188 being common to all three samples (Supplementary Figure 1). Thus, most hemocyte markers expressed in larval blood cells are also expressed in the adult. Notably, 11 of these hemocyte markers (Col4a1/Cg25C, Ppn, Nplp-2, Pxn, Fer2LHC, Fer1HCH, PPO2, PPO1, crq, Sr-Cl and Glt) were among the 60-most strongly expressed genes in adult blood cells (Supplementary Table 2). In contrast, $\mathrm{gcm}$ and $\mathrm{gcm} 2$, which were shown to be expressed in embryonic but not in larval blood cells (AvetRochex et al., 2010; Bazzi et al., 2018; Ramond et al., 2020), were not detected in adult (or larval) hemocyte RNA-seq samples (Supplementary Tables 2-4).

Despite expressing many genes in common, adult and larval hemocytes also exhibit distinct features. Indeed, principal component analysis showed that $77 \%$ of gene expression variance among samples was accounted by stage differences (adult versus larva) (Figure 2B). Furthermore, differential gene expression analysis with DESeq2 revealed that 1074 genes were differentially expressed between adult blood cells and both larval peripheral hemocytes and lymph glands (adjusted $p$-value $<0.01$ and fold change $>2$ ), with 864 genes up-regulated and 210 downregulated in adult hemocytes (Figures 2C,D and Supplementary Table 6). For comparison, 330 genes were differentially expressed between larval peripheral hemocytes and both adult blood cells and lymph glands (151 up-regulated, 179 down-regulated), and 218 genes were differentially expressed between lymph glands and both adult and larval peripheral hemocytes ( 99 up-regulated, 119 down-regulated). For instance, consistent with previous immunostaining results (Kurucz et al., 2007), we observed that Hemese $(\mathrm{He}$ ) expression was down-regulated in adult hemocytes as compared to larval ones (Supplementary Table 6). In addition, to validate these results, we performed RT-qPCR on several genes that were differentially expressed between adult and larval blood 


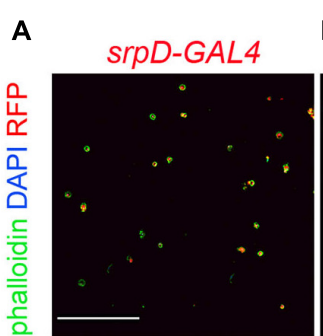

5 days

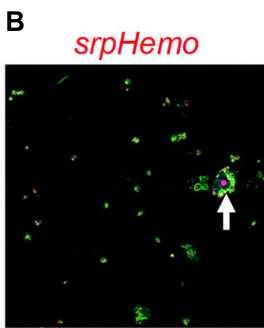

2 days

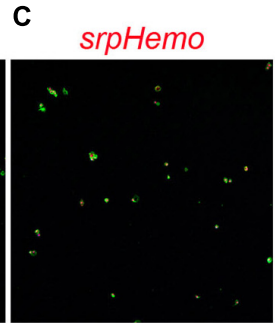

5 days

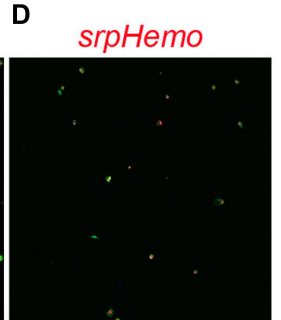

35 days
E

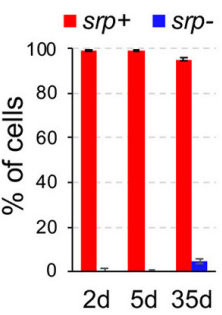

$\mathbf{F}$

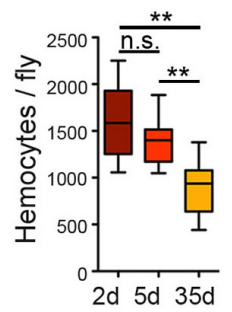

FIGURE 1 | Recovery of Drosophila blood cell by bleeding. (A-D) Confocal images of cells recovered from srpD-GAL4,UAS-mCherry (A, srpD-GAL4) or srpHemo-His2A-RFP (B-D, srpHemo) adult females. Cells were counterstained with phalloidin (green) and DAPI (blue). The age of the flies is indicated below each panel. Scale bar: $200 \mu \mathrm{m}$. The arrow in panel (B) indicates a fat body cell surrounded by hemocytes. (E) Quantifications of the proportion of cells (DAPI+) expressing srpHemo-His2A-RFP according to fly age. Means and standard deviations of five independent experiments are shown. A total of 2316,2351 , and 1516 cells were counted in 2- day-, 5- day-, and 35-day-old flies, respectively. (F) Quantifications of the absolute number of cells recovered per fly at different ages. Means and standard deviations from eight independent experiments are represented. Kruskal-Wallis test was used for statistical analysis. n.s., non-significant ( $p>0.05)$; ${ }^{* *} p<0.01$.

cells using independent RNA samples. Thereby, we confirmed that $h s p 23$, Tig, mthl6 and CG15115 were downregulated in adult hemocytes while ninaD, Fhos, Tps1, Cyp12a4, Ald and Men were up-regulated (Figure 2E). Furthermore, reporter lines for NimC4 and $M p$ also showed that these two genes were expressed in adult but not in larval hemocytes (Figures 2F,G).

Gene Ontology enrichment analyses underlined the upregulation of genes implicated in small molecule metabolism processes, including ATP metabolism (as seen with the overexpression of several enzymes involved in glycolysis, TCA cycle, or oxidative phosphorylation) and a down-regulation of genes regulating chromatin organization in adult hemocytes (Table 1). Along the same line, Gene Ontology analysis considering the 1000 most strongly expressed genes in larval, lymph gland or adult blood cells revealed shared features between them, with for instance a strong over-representation for genes implicated in translation, vesicle-mediated transport or immune response, but also confirmed the singular enrichment for ATP metabolic processes among highly expressed genes in adult hemocytes (Table 2).

Surprisingly, one of the top-enriched genes in adult hemocytes is NimC4, which was recently shown to be highly enriched in stage 16 embryonic hemocytes as compared to larval descendants (i.e., larval peripheral hemocytes) in a comparative transcriptomic analysis (Cattenoz et al., 2020). Moreover, reminiscent of the situation that we observed, embryonic and their larval progenies were found to have distinct metabolic gene signature. We thus reanalyzed the RNA-seq data from Cattenoz et al. (2020) using the same settings as for our transcriptomes. Thereby, we established a list of 4892 differentially expressed genes between embryonic and peripheral hemocytes (adjusted $p$-value $<0.01$ and fold change $>2$; Supplementary Table 7) that we compared with our results. Strikingly, $49 \%$ (422/864) of the genes up-regulated in adult versus larval hemocytes are also over-expressed in embryonic blood cells as compared to larval blood cells (2.9-fold over-enrichment, $p<4.8 \mathrm{E}$ 113) (Figure 2H). Similarly, 46\% (97/210) of the genes downregulated in adult versus larval hemocytes are also repressed in embryonic blood cells (4.2-fold over-enrichment, $p<1.3 \mathrm{E}-38$ ) (Figure 2H). The common over-expressed genes in embryonic and adult hemocytes versus larval ones were implicated in small molecule/ATP metabolic processes, while down-regulated genes were involved in cell cycle (Table 3). Hence, many genes and processes differentially regulated between adult and larval hemocytes are similarly regulated between embryonic and larval hemocytes.

In sum, these data show that adult hemocytes share a significant portion of their transcriptome with larval blood cells but also exhibit distinct features, some of which appear to be shared with embryonic hemocytes.

\section{Characterization of the Adult Hematopoietic System Landscape}

To better define the cellular composition of the adult hematopoietic system, we then analyzed the expression of a series of well-characterized reporters classically used to study embryonic and larval blood cell types (see the section "Materials and Methods").

First, we assessed the expression of plasmatocyte and crystal cell differentiation markers. In 5-day-old flies, the plasmatocyte reporters for Peroxidasin (Pxn), Collagen 25C (Cg25C, also known as Collagen type IV alpha1), Hemolectin ( $\mathrm{Hml})$, and croquemort (crq) were expressed in $\sim 95 \%, 90 \%, 65 \%$, and $25 \%$ of the blood cells, respectively, while those for the crystal cell markers Black cells (Bc, also known as Prophenoloxidase 1, PPO1) and lozenge $(l z)$ were expressed in 8 to $12 \%$ of the cells (Figures $3 \mathrm{~A}-\mathrm{E}, \mathbf{G}$ and Supplementary Figures 2A-F). The proportion of hemocytes expressing these reporters was similar in 35-day-old flies, except for a slight but significant decline in the proportion of $\mathrm{Pxn}^{+}$ cells (Figure 3G). The analysis of double transgenic lines revealed that (almost) all the cells expressing $\mathrm{Hml}$ co-expressed $\mathrm{srp}$ and Pxn (Figures 3H-H",K-K",Q). Besides, $\sim 90$ of the $\mathrm{crq}^{+}$cells were $\mathrm{Hml}^{+}$(Figures 30-O",Q). Among the $\mathrm{Bc}^{+}$cells, 100\% expressed srp and Pxn, $\sim 90 \% \mathrm{Cg} 25 \mathrm{C}$ and $60 \% \mathrm{Hml}$ (Figures 3II",L-L",N-N",P-P",Q). Of note, it was previously observed 
A

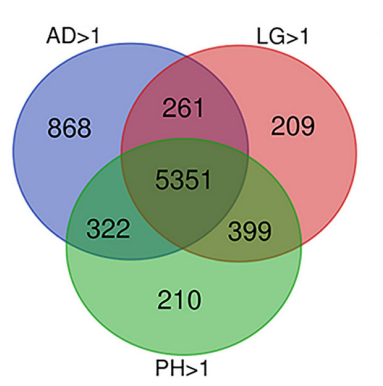

D

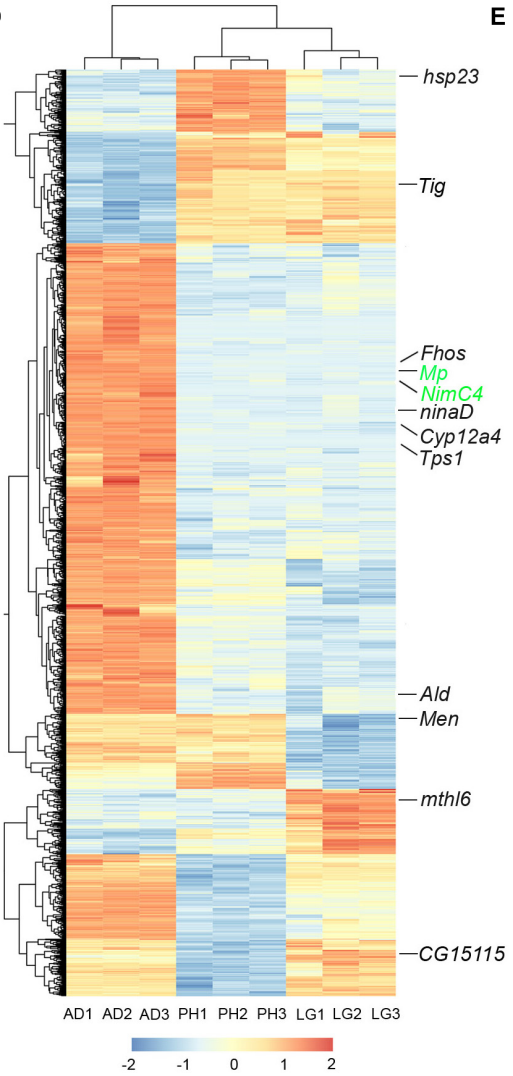

B

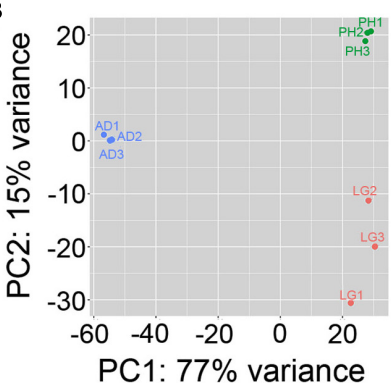

C

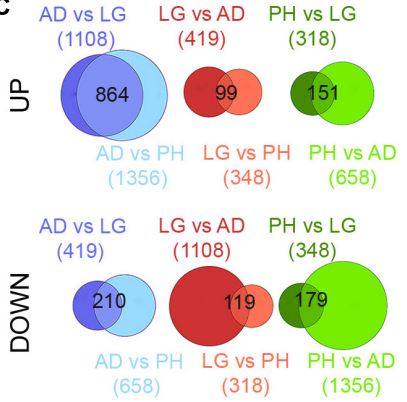

F

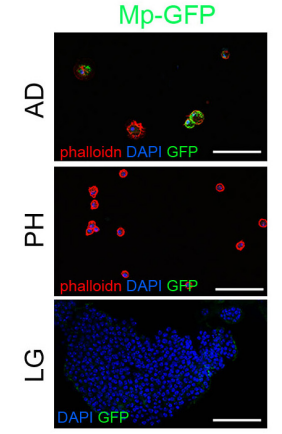

G

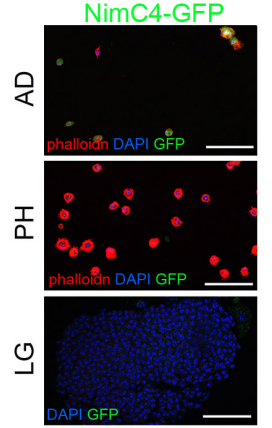

H

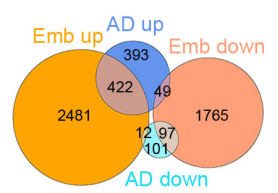

FIGURE 2 | Analysis of adult blood cell gene expression profile. (A) Venn diagrams showing the number of genes expressed (RPKM $>1$ in all three biological replicates) in adult hemocytes (AD), larval peripheral hemocytes $(\mathrm{PH})$ and larval lymph glands (LG) as determined by RNA-seq on $w^{1118}$ females. (B) Principal component analysis of the RNA-seq profiles of adult, peripheral and lymph gland biological triplicates. (C) Venn diagrams showing the overlaps between differentially expressed genes in pairwise comparisons (adjusted $p$-value $<0.01$ and fold change $>2$ ). Upper panels: up-regulated genes. Lower panels: down-regulated genes. The number of up- or down-regulated genes in each condition is indicated in brackets and the number of genes commonly deregulated is indicated in the Venn diagram intersection. (D) Heat map of the genes differentially expressed between adult versus larval hemocytes. The genes tested in panels $\mathbf{E}$ to $\mathbf{G}$ are indicated. (E) The expression level of the indicated genes was determined by RT-qPCR on adult hemocytes, larval peripheral hemocytes and larval lymph glands. Expression levels were normalized to rp49. Means and SEM from three independent experiments are represented. (F,G) Confocal images showing GFP expression in adult and larval hemocytes from Mp-GFP (F) or NimC4-GFP (G) reporter lines. Nuclei were stained with DAPI (blue). For adult and larval peripheral hemocytes (upper and middle panels), the samples were stained with phalloidin (red). Lower panels show a lymph gland anterior lobe. Scale bar: $50 \mu \mathrm{m}$. (H) Venn diagrams showing the overlaps of up- or down-regulated genes between adult versus larval hemocytes (AD, blue) or embryonic versus larval hemocytes (Emb, orange).

that the "plasmatocyte" markers $\mathrm{Cg} 25 \mathrm{C}, \mathrm{Pxn}$ and $\mathrm{Hml}$ are also expressed in larval crystal cells (Jung et al., 2005). We did not observe significant shifts in the repartition of these populations between 5 - and 35-day-old flies, indicating that the overall composition of the adult hematopoietic system remains stable during aging (Figure 3Q).
Next, we tested whether lamellocytes could be retrieved in the adult. Intriguingly, we found that $m s n F 9$ reporter, whose activity is induced in lamellocytes in the larvae (Tokusumi et al., 2009b), was expressed in $\sim 92 \%$ of the adult hemocytes (Supplementary Figure 2G). However, we did not observe any cell with high actin content and/or expressing the $\beta$-integrin Myospheroid 
TABLE 1 | Main gene ontology terms enriched in genes over- (up AD) or under(down AD) expressed in adult as compared to larval hemocytes.

\begin{tabular}{|c|c|c|c|}
\hline GO-term & \multicolumn{2}{|c|}{ Up AD } & Down AD \\
\hline Small molecule metabolic process & \multicolumn{2}{|c|}{$5.06 \mathrm{E}-42(n=158)$} & \\
\hline ATP metabolic process & \multicolumn{2}{|c|}{$1.66 \mathrm{E}-39(n=63)$} & \\
\hline Inner mitochondrial membrane protein & \multicolumn{2}{|c|}{$3.50 \mathrm{E}-30(n=52)$} & \\
\hline Oxidoreductase activity & \multicolumn{2}{|c|}{$7.74 \mathrm{E}-29(n=113)$} & \\
\hline Nucleosome & \multicolumn{3}{|c|}{$6.27 \mathrm{E}-53(n=45)$} \\
\hline Chromatin organization & \multicolumn{3}{|c|}{$5.46 \mathrm{E}-29(n=51)$} \\
\hline \multicolumn{4}{|c|}{$p$-Values and gene numbers are indicated for each category. } \\
\hline \multicolumn{4}{|c|}{$\begin{array}{l}\text { TABLE } 2 \text { | Main gene ontology terms among the } 1000 \text { most strongly expressed } \\
\text { genes in adult hemocytes (AD), peripheral hemocytes }(P H) \text { and lymph glands (LG). }\end{array}$} \\
\hline GO-term & $\begin{array}{l}\text { AD top } \\
1000\end{array}$ & $\begin{array}{l}\text { PH top } \\
1000\end{array}$ & $\begin{array}{l}\text { LG top } \\
1000\end{array}$ \\
\hline Cytoplasmic translation & $\begin{array}{l}1.50 \mathrm{E}-71 \\
(n=89)\end{array}$ & $\begin{array}{l}2.33 \mathrm{E}-70 \\
(n=88)\end{array}$ & $\begin{array}{c}3.53 \mathrm{E}-77 \\
(n=93)\end{array}$ \\
\hline $\begin{array}{l}\text { Generation of precursor } \\
\text { metabolites }\end{array}$ & $\begin{array}{c}6.91 \mathrm{E}-42 \\
(n=83)\end{array}$ & $\begin{array}{l}1.20 \mathrm{E}-04 \\
(\mathrm{n}=30)\end{array}$ & $\begin{array}{l}3.67 \mathrm{E}-04 \\
(\mathrm{n}=24)\end{array}$ \\
\hline ATP metabolic process & $\begin{array}{l}1.61 \mathrm{E}-41 \\
(n=156)\end{array}$ & $\begin{array}{l}1.89 E-06 \\
(n=28)\end{array}$ & $\begin{array}{c}8.22 \mathrm{E}-07 \\
(n=27)\end{array}$ \\
\hline $\begin{array}{l}\text { Structural constituent of } \\
\text { ribosome }\end{array}$ & $\begin{array}{c}2.79 \mathrm{E}-31 \\
(n=80)\end{array}$ & $\begin{array}{l}6.00 \mathrm{E}-28 \\
(n=75)\end{array}$ & $\begin{array}{c}4.48 \mathrm{E}-31 \\
(n=79)\end{array}$ \\
\hline Oxidoreductase activity & $\begin{array}{l}4.19 \mathrm{E}-17 \\
(n=105)\end{array}$ & $\begin{array}{l}2.77 \mathrm{E}-04 \\
(\mathrm{n}=67)\end{array}$ & $\begin{array}{l}1.29 E-03 \\
(n=64)\end{array}$ \\
\hline Vesicle-mediated transport & $\begin{array}{c}2.44 \mathrm{E}-14 \\
(n=86)\end{array}$ & $\begin{array}{c}2.13 \mathrm{E}-18 \\
(n=94)\end{array}$ & $\begin{array}{c}6.25 \mathrm{E}-12 \\
(n=81)\end{array}$ \\
\hline Phagocytosis & $\begin{array}{c}8.43 \mathrm{E}-09 \\
(n=18)\end{array}$ & $\begin{array}{l}7.54 \mathrm{E}-09 \\
(n=18)\end{array}$ & $\begin{array}{c}2.52 E-04 \\
(n=12)\end{array}$ \\
\hline Immune system process & $\begin{array}{l}1.48 \mathrm{E}-05 \\
(n=56)\end{array}$ & $\begin{array}{c}2.72 \mathrm{E}-07 \\
(n=61)\end{array}$ & $\begin{array}{c}2.17 \mathrm{E}-07 \\
(n=62)\end{array}$ \\
\hline
\end{tabular}

p-Values and gene numbers are indicated for each category. Categories particularly enriched in adult hemocytes are highlighted in bold.

(Mys), two characteristic features of lamellocytes (Irving et al., 2005; Supplementary Figures 3C,I), suggesting that lamellocytes are not present and that the $m s n F 9$ reporter is constitutively active in adult blood cells. Since lamellocytes are absent in healthy larvae but massively induced in response to infection by eggs of the parasitoid wasp Leptopilina boulardi (Lanot et al., 2001), we assessed their presence in adult flies that survived wasp infestation. Still, $\beta$-integrin and phalloidin staining did not reveal the presence of lamellocytes in infected adults, while they were readily recovered in bleeds from infected larvae (Supplementary Figures 3B,D,H,J). Furthermore, when using flies carrying the gain-of-function Hop $T^{\text {Tum-l }}$, which exhibit constitutive activation of the JAK kinase Hopscotch leading to lamellocyte production in the larvae (Luo et al., 1995), we did not observe lamellocytes in the adult either (Supplementary Figures 3E,F,K,L). Thus, it appears that lamellocytes do not persist during adulthood.

Finally, to reveal the presence of putative blood cell progenitors, we made use of tepIV-GAL4, Ance-GFP, dome PG125 $_{\text {- }}$ GAL4 and domeMeso-GAL4, all of which have been shown to label larval prohemocytes in the lymph gland (Krzemien et al., 2007; Avet-Rochex et al., 2010; Louradour et al., 2017; Cho
TABLE 3 | Main gene ontology terms enriched in genes up- or down-regulated in adult (AD) and embryonic (Emb) hemocytes as compared to larval hemocytes.

\begin{tabular}{llc}
\hline GO-term & Up AD and Emb & Down AD and Emb \\
\hline Small molecule metabolic process & $2.48 \mathrm{E}-24(n=83)$ & \\
ATP metabolic process & $4.21 \mathrm{E}-22(n=34)$ & \\
Respiratory chain complex & $3.07 \mathrm{E}-21(n=26)$ & \\
Oxidoreductase activity & $1.37 \mathrm{E}-15(n=58)$ & \\
Cell cycle & & $9.73 \mathrm{E}-8(n=20)$ \\
\hline p-Values and gene numbers are indicated for each category. &
\end{tabular}

et al., 2020; Rodrigues et al., 2021) or in peripheral blood cells (Fu et al., 2020). While we did not detect any expression of tepIV-GAL4, Ance-GFP or dome $e^{P G 125}$-GAL4 in adult hemocytes (Supplementary Figures $\mathbf{2 H}-\mathbf{J}$ ), we found that domeMeso-GAL4 was expressed in $\sim 4 \%$ of cells in both 5 - and 35 -day-old flies (Figures 3F,G). domeMeso was expressed in cells exhibiting a characteristic fusiform shape, with one or two filopodial extension, but not in round hemocytes (Figures 3F,J-J"',M-M"'). Importantly, none of the fusiform cells expressed the blood cell differentiation markers tested above ( $P x n, C g 25 C, H m l, c r q, B c$, $l z$ and $m s n)$, but they expressed $s r p$, albeit at lower level than the other blood cells (Figures 3H"'-P'” and Supplementary Figures 4A-H). Also, immunostaining against the plasmatocyte marker $\mathrm{P} 1 / \mathrm{NimC1}$ as well as in situ hybridization against Pxn or against the crystal cell marker $B c / P P O 1$ confirmed that domeMeso ${ }^{+} /$fusiform cells did not express these blood cell differentiation markers (Supplementary Figures 4I-K), strongly suggesting that they could be blood cell progenitors.

In sum, our data indicate that the adult hematopoietic system is essentially composed of plasmatocytes $(\sim 85 \%)$ and crystal cells (8 to 10\%) and could harbor a small population of undifferentiated cells $(\sim 4 \%)$. Furthermore, as schematized in Figure 3R, adult blood cells can be classified into different subcategories (depending on the expression of Pxn, Hml, crq, Bc and domeMeso) whose proportions are relatively stable between young and old flies.

\section{Abdominal Hematopoietic Hubs Have a Similar Composition as Adult Bleeds}

Since adult bleeds may not accurately reflect the composition of the adult hematopoietic system, we also assessed the expression of some of the above reporters in the dorsal abdomen. As shown in Figures 4A-G,A'-G' srp, Pxn, Cg25C, Hml, crq, Bc, or domeMeso expression was observed as dispersed cells in the abdomen, especially in segments A1 to A3 along the heart, in the socalled "hematopoietic hubs." srp expression was also prominent in pericardial cells (Figure $\mathbf{4 A}$, arrows). In the hematopoietic hubs, $\mathrm{Hml}^{+}$cells represented $65 \%$ of the hemocytes as judged by co-labeling with srp or Pxn and no $\mathrm{Hml}^{+} \mathrm{srp}^{-}$or cells $\mathrm{Hml}^{+}$ $\mathrm{Pxn}^{-}$were observed (Figures $\mathbf{4 H}-\mathbf{H}^{\prime}, \mathbf{K}-\mathbf{K}$ ”,P-P”). Crystal cells $\left(\mathrm{BC}^{+}\right)$and putative prohemocytes (domeMeso ${ }^{+}$) represented $\sim 10 \%$ and $\sim 3 \%$ of the hemocytes, respectively (Figures $4 \mathrm{I}$ I",J-J",P-P"). $90 \%$ of the crystal cells expressed Cg25C and $55 \%$ of them expressed $\mathrm{Hml}$ (Figures 4M-M",O-O”,P-P”). All domeMeso ${ }^{+}$cells expressed srp at low levels and none expressed 


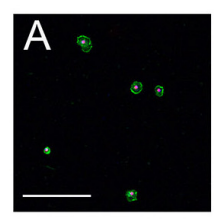

Pxn-RFP

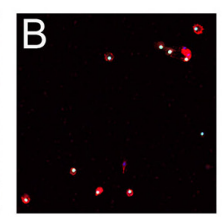

Cg25C-GFP

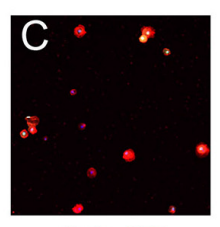

$H m I \triangle>G F P$

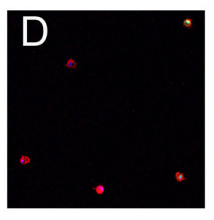

crq $>$ GFP

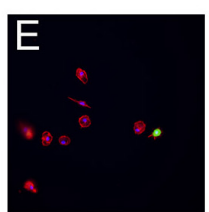

$B C-G F P$

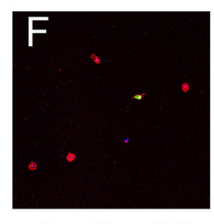

domeMeso $>$ GFP

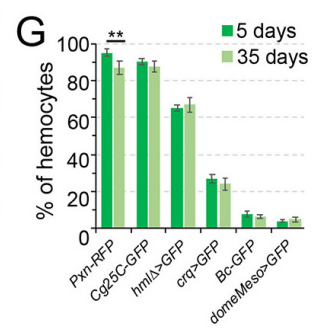

- $\mathrm{G}-\mathrm{R}+\quad \mathrm{G}+\mathrm{R}+\quad \mathrm{G}+\mathrm{R}-$
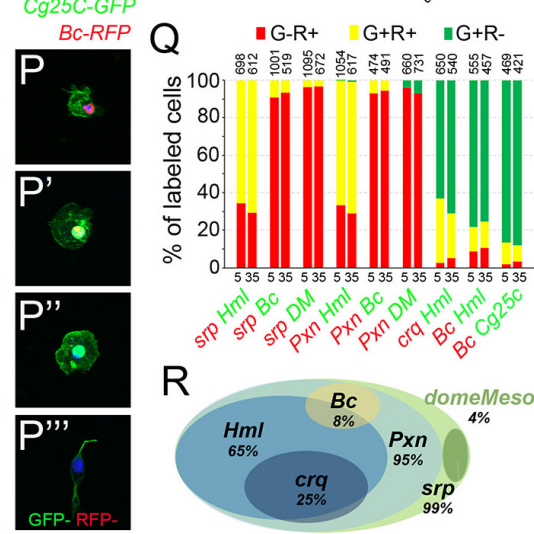

FIGURE 3 | Analysis of blood cell transgenic markers in adult bleeds. (A-F) Confocal views showing representative bleeds from 5-day-old flies carrying the following transgenes: Pxn-RedStinger (A, Pxn-RFP), Cg25C-GFP (B), Hml $\Delta$-GAL4,UAS-2xEYFP (C, HmI $>$ > GFP), crq-GAL4,UAS-2xEYFP (D, crq > GFP), BcF2-GFP (E, Bc-GFP), domeMeso-GAL4,UAS-2xEYFP (F, domeMeso> GFP). Cells were counterstained with phalloidin (A: green, B-F: red) and DAPI (blue). Scale bar 200 $\mu$ m. (G) Quantifications of blood cell proportions expressing the indicated transgenes in 5- or 35-day-old flies. Means and standard deviations from four to six independent experiments are represented. From 200 to 600 DAPI+ cells were scored in each experiment. ${ }^{\star *} p<0.01$ (Student's $t$-test). (H-P) High magnification views showing the different types of blood cells observed in bleeds from 5-day-old flies carrying the following combination of transgenes: srpHemo-His2A-RFP, HmI $\triangle$-GAL4,UAS-2xEYFP (H), srpHemo-His2A-RFP, BcF2-GFP (I), srpHemo-His2A-RFP, domeMeso-GAL4,UAS-2xEYFP (J), Pxn-RedStinger, HmI - -GAL4,UAS-2xEYFP (K), Pxn-RedStinger, BcF2-GFP (L), Pxn-RedStinger, domeMeso-GAL4, UAS-2xEYFP (M), HmI - -GAL4,UAS-2xEYFP, BcF6-mCherry (N), HmI $\Delta$-GAL4,UAS-2xEYFP, crq-RedStinger (0), Cg25C-GFP, BcF6-mCherry (P). GFP and RFP signals are nuclear; cells were counterstained with DAPI (blue) and phalloidin (green). The top three panels show round cells expressing or not RFP and/or GFP; the bottom panels ("') show fusiform cells together with their GFP/RFP expression status. Scale bar $20 \mu \mathrm{m}$. (Q) Quantifications of the proportion of cells expressing the indicated combination of markers in 5- or 35-day-old flies. Mean values from at least three independent experiments are presented. No significant difference (Student's $t$-test $p<0.05$ ) was observed in the proportion of $\mathrm{GFP}^{+} \mathrm{RFP}^{+}, \mathrm{GFP}^{+} \mathrm{RFP}^{-}$or GFP ${ }^{-} \mathrm{RFP}^{+}$blood cell populations between 5- and 35-day-old flies. The total numbers of GFP ${ }^{+}$and/or RFP+ cells scored are indicated at the top of each column. (R) Schematic representation of the adult hematopoietic landscape. The proportion of cells expressing each marker is indicated.

Pxn (Figures 4J-J”,L-L”,P-P”). Similar observations were made when analyzing the expression of these markers in 35-dayold flies and we did not observe significant differences in the proportion of each blood cell populations between 5-day- and 35-day-old flies (Figure 4P). These results are consistent with our observations on adult bleeds and indicate that the adult hematopoietic system is composed of different subpopulations of plasmatocytes and crystal cells as well as a few putative blood cell progenitors.

\section{Hemolectin Is Expressed in a Sub-Population of Adult Hemocytes}

The $\mathrm{Hml}$ driver has been used in several instances to manipulate gene expression in adult blood cells and/or test the function of these cells, notably by generating "hemoless" adults through the expression of an apoptosis inducer in blood cells (Charroux and Royet, 2009; Defaye et al., 2009; Ayyaz et al., 2015; Ghosh et al., 2015; Woodcock et al., 2015; Sanchez Bosch et al., 2019). Yet, our results suggest that this driver is not active in every adult hemocyte but rather labels a specific subpopulation. To confirm this hypothesis, we expressed the apoptotic inducer Bax under the control of $\mathrm{Hml}$ either during the whole development or only from day 1 of adulthood by using a tubGAL-80 ts transgene to restrict its expression temporally, and we assessed the presence of hemocytes in 10-day-old adult flies. $\mathrm{Hml}$-driven expression of Bax specifically during adulthood caused an almost total ablation of $\mathrm{Hml}^{+}$blood cell population as compared to control flies (Figures 5A-A"',C-C'”), but Pxn ${ }^{+}$cells were still present in adult bleeds and abdominal preparations (Figures 5B-B"',DD"'). When Bax was expressed from embryogenesis onward, we observed an efficient ablation of $\mathrm{Hml}^{+}$cells and very few $\mathrm{Pxn}^{+}$blood cells in adult abdominal hubs but, in contrast with the normal situation (Figures 5E-E"',G-G”'), we retrieved many $\mathrm{Pxn}^{-}$cells when these individuals were bled (Figures 5FF','H-H'”). While some of these $\mathrm{Pxn}^{-}$cells were fusiform, as in the control, most had the typical round morphology of differentiated hemocytes, suggesting that the chronic expression of Bax in $\mathrm{Hml}^{+}$cells leads to the repression of Pxn. Consistent with this hypothesis, immunostaining against $\mathrm{P} 1 / \mathrm{NimC} 1$ showed 

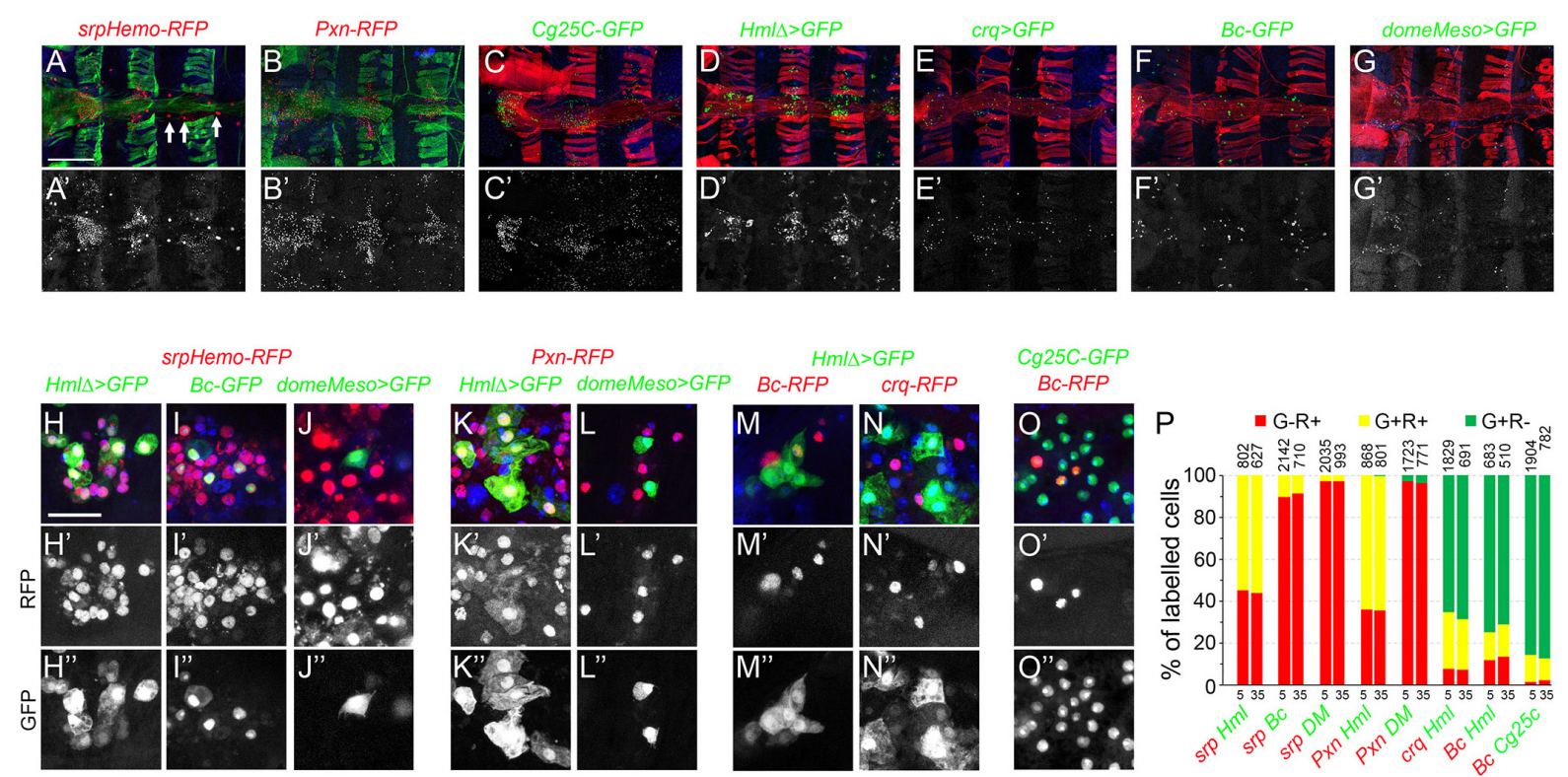

FIGURE 4 | Analysis of blood cell transgenic markers in the adult abdomen. (A-F) Confocal views of the abdominal segments A1 to A4 of 5-day-old females carrying the following transgenes: srpHemo-His2A-RFP (A), Pxn-RedStinger (B), Cg25C-GFP (C), Hml $\triangle$-GAL4,UAS-2xEYFP (D), crq-GAL4,UAS-2xEYFP (E), BCF2-GFP (F), domeMeso-GAL4,UAS-2xEYFP (G). Cells were counterstained with phalloidin (A,B: green; C-G: red) and DAPI (blue). Arrows in panel (A) indicate pericardial cells expressing srpHemo. The lower panels show only the transgene expression. Scale bar $200 \mu \mathrm{m}$. (H-O) High magnification views of hemocytes in the abdominal hematopoietic hubs (heart region in A1 or A2 segments) of 5-day-old females carrying the following transgenes: srpHemo-His2A-RFP, HmI $\triangle$-GAL4,UAS-2xEYFP (H), srpHemo-His2A-RFP, BcF2-GFP (I), srpHemo-His2A-RFP, domeMeso-GAL4,UAS-2xEYFP (J), Pxn-RedStinger, HmI $\Delta$-GAL4,UAS-2xEYFP (K), Pxn-RedStinger, domeMeso-GAL4,UAS-2xEYFP (L), HmI $\Delta$-GAL4,UAS-2xEYFP, BcF6-mCherry (M), HmI $\Delta$-GAL4,UAS-2xEYFP, crq-RedStinger (N), Cg25C-GFP, BcF6-mCherry (O). The lower panels show only the red (H'-O') or the green (H'-O') channel. Nuclei were stained with DAPI (blue). Scale bar 50 $\mu$ m. (P) Quantifications of the proportion of cells expressing the indicated combination of markers in 5- or 35-day-old flies. Mean values from at least three independent experiments are presented. No significant difference (Student's $t$-test $p<0.05$ ) was observed in the proportion of GFP ${ }^{+}$RFP $^{+}, \mathrm{GFP}^{+} \mathrm{RFP}^{-}$or GFP- RFP ${ }^{+}$blood cell populations between 5- and 35-day-old flies. The total numbers of GFP ${ }^{+}$and/or RFP ${ }^{+}$cells scored are indicated at the top of each column.
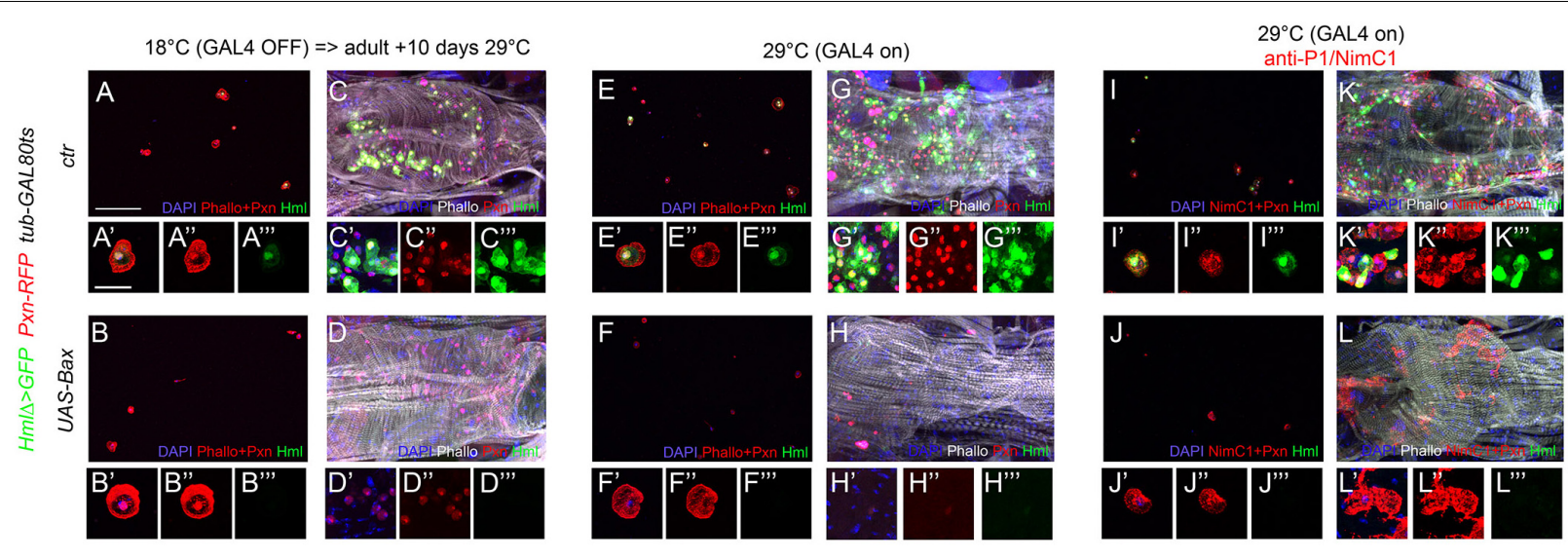

FIGURE 5 | Adult blood cells are still present following the ablation of $\mathrm{Hml}^{+}$hemocytes. Confocal views of adult bleeds (A,B,E,F,I,J) and abdominal hubs (C,D,G,H,K,L) from tub-GAL80ts, Pxn-RedStinger, Hm/ - -GAL4,UAS-2xEYFP (A,C,E,G,I,K) and tub-GAL80ts, Pxn-RedStinger, Hm/ $\triangle$-GAL4,UAS-2xEYFP, UAS-Bax (B,D,F,H,J,L) flies expressing (or not) Bax in $\mathrm{Hm}^{+}{ }^{+}$cells only during adulthood (A-D) or throughout their life (E-L). Cells were stained with phalloidin (A,B,E,F: red; C,D,G,H,K,L: white; I,J: no phalloidin) and nuclei with DAPI (blue). (I-L): immunostaining against P1/NimC1 (red). Lower panels show high magnification views of hemocytes. (A'-L') Merged blue, red and green channels, (A"'-L'): red channel only, (A'"'-L'”): green channel only. Upper panels: scale bar $200 \mu \mathrm{m}$; lower panels: scale bar $20 \mu \mathrm{m}$.

that many cells expressing this plasmatocyte marker were still present in adult bleeds and abdominal preparations following the constitutive expression of Bax in $\mathrm{Hml}^{+}$cells (Figures 5J-J"',L-L"' and Supplementary Figure 5). These results show that killing $\mathrm{Hml}^{+}$blood cells is not sufficient to generate "hemoless" flies and might alter the gene expression program of the remaining blood 


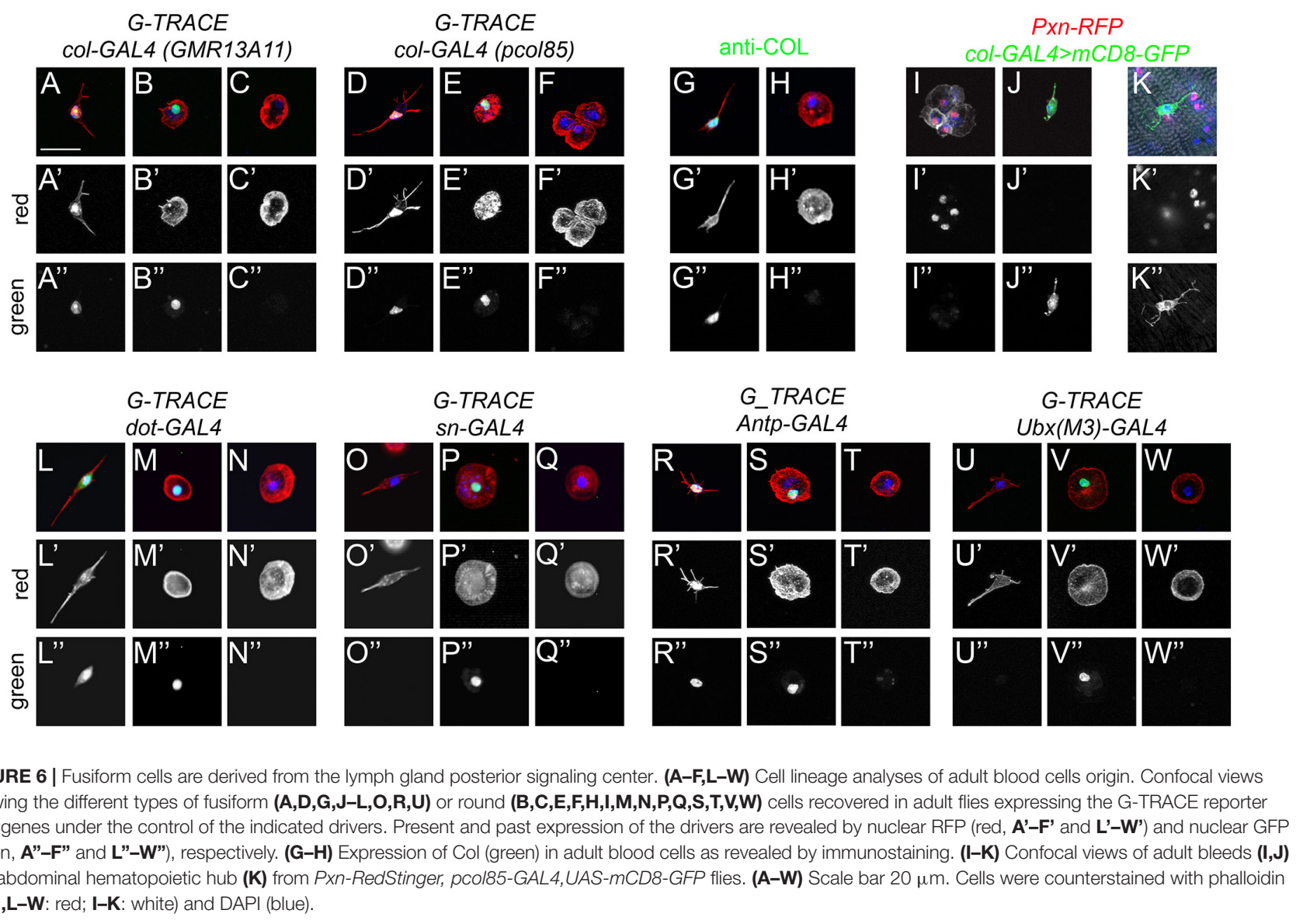

cells. These data also indicate that during adulthood $\mathrm{Hml}^{+} \mathrm{Pxn}^{+}$ and $\mathrm{Hml}^{-} \mathrm{Pxn}^{+}$cells form two distinct populations and that fusiform cells are not derived from $\mathrm{Hml}^{+}$cells.

\section{domeMeso+ "Undifferentiated Hemocytes" Are Not Derived From Larval Blood Cell Progenitors but From the Lymph Gland Posterior Signaling Center}

A previous report showed that a small fraction of cells expressing Srp did not express the blood cell differentiation markers P1/NimC1 (for plasmatocytes) or Hindsight (for crystal cells), suggesting that they could be hematopoietic progenitors (Ghosh et al., 2015). In addition, these cells were proposed to derive from the posterior lobes of the larval lymph gland based on lineage tracing experiments using the col-GAL4 driver GMR13A11. While col-GAL4-derived cells from the larval lymph gland were shown to contribute to adult plasmatocytes and crystal cells (Ghosh et al., 2015), it remains to demonstrate that these cells can proliferate and/or differentiate during adulthood and thus constitute a genuine adult hematopoietic progenitor population. Our results above strongly suggested that fusiform/domeMeso ${ }^{+}$cells could correspond to this putative hematopoietic progenitor population. To test this hypothesis, we first assessed their origin. Using the G-TRACE cell lineage tracing technique (Evans et al., 2009) with either GMR13A11 or the classically used pcol85 col-GAL4 line, we found that all fusiform cells belonged to the col lineage-traced cells (Figures 6A,D), indicating that they correspond to the previously described adult blood cell progenitors. Interestingly, we found that these cells maintain col driver expression (Figures 6A-A",D-D"), while we only observed past/lineage-traced expression in round hemocytes (Figures 6B-B",E-E"). This result was confirmed by immunostaining, which revealed that $\mathrm{Col}$ was expressed in fusiform/domeMeso ${ }^{+}$cells, but not in other adult blood cells (Figures 6G-G",H-H" and Supplementary Figures 6A,B). Furthermore, in accordance with the above characterization of domeMeso ${ }^{+}$cells, $\mathrm{Col}^{+}$cells were $\mathrm{Pxn}^{-}$both in adult bleeds and in abdominal hematopoietic hubs (Figures 6J-J",K-K").

These results are thus consistent with the hypothesis that $\mathrm{col}^{+}$progenitors could give rise to differentiated hemocytes. Importantly though, the col-GAL4 lines pcol85 and GMR13A11 are not only expressed in blood cell progenitors present in the lymph gland posterior lobes but also in the PSC (Benmimoun et al., 2012; Ghosh et al., 2015; Rodrigues et al., 2021). Moreover, recent single-cell RNA-seq analyses revealed that PSC-like cells expressing col and Antp are present in circulating larval blood cells (Cattenoz et al., 2021). Hence, these putative adult hematopoietic progenitors could have diverse origins. First, to test whether they were derived from the 


\section{domeMeso>EYFP}
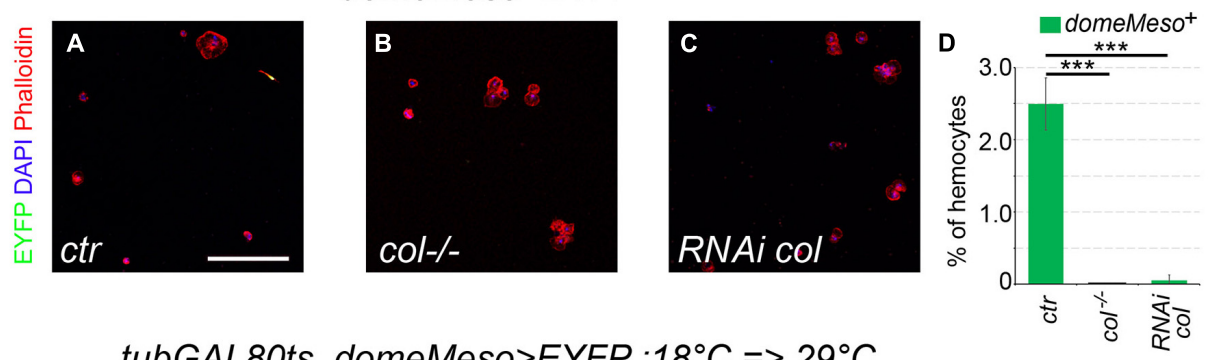

tubGAL80ts, domeMeso $>$ EYFP $: 18^{\circ} \mathrm{C}=>29^{\circ} \mathrm{C}$
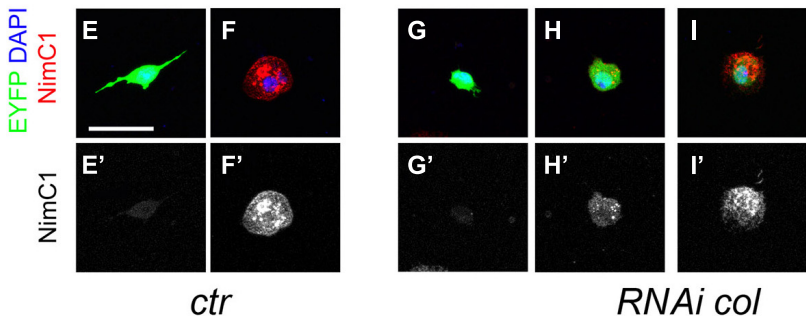

RNAi col

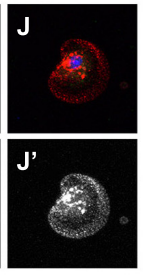

K DM high/fusiform

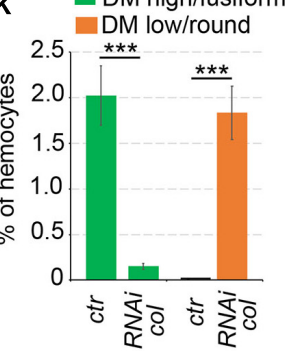

tubGAL80ts, domeMeso>G-TRACE
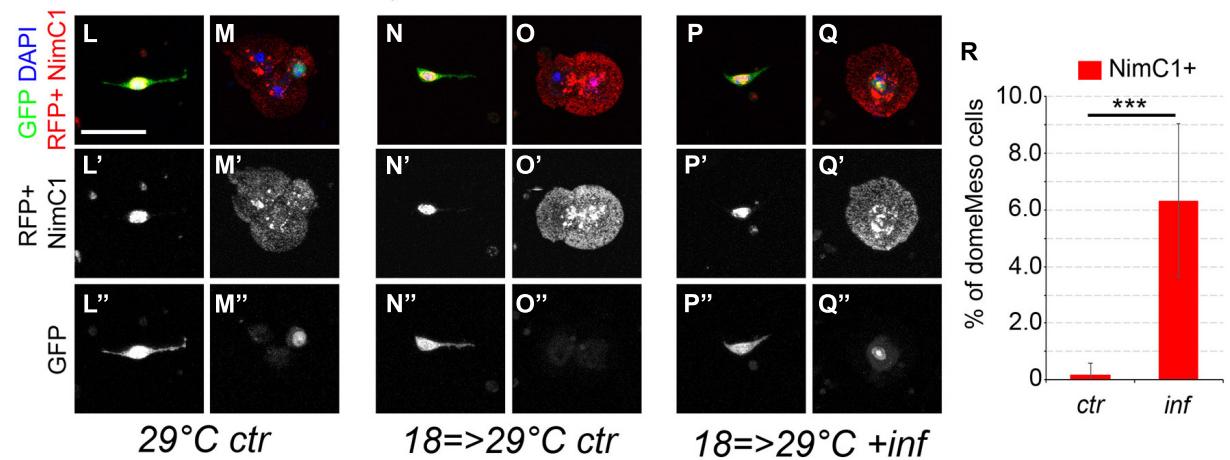

srp $>F U C C I$ (GFP-E2F, RFP-nlsCycB)

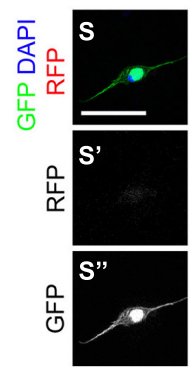

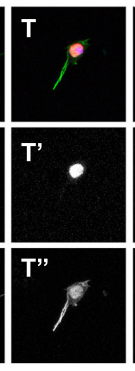

ctr

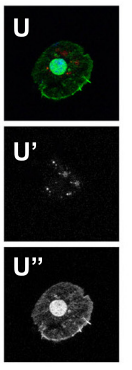

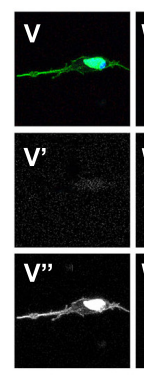

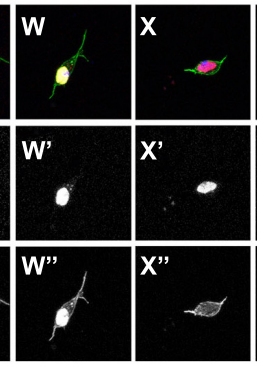

infected
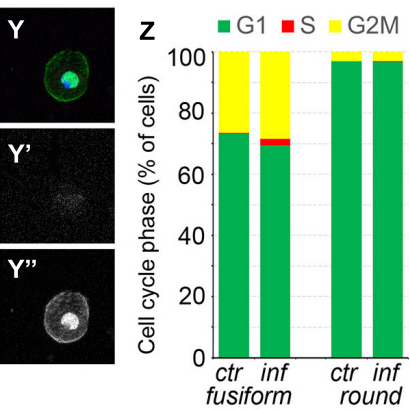

FIGURE 7 | Fusiform cells can differentiate and proliferate. (A-C) Confocal views of blood cells from domeMeso-GAL4, UAS-2xEYFP (A, ctr), col-/-, domeMeso-GAL4, UAS-2xEYFP (B, col-/-) and domeMeso-GAL4, UAS-2xEYFP, UAS-RNAi col (C, RNAi col) adult flies. Blood cells were stained with phalloidin (red) and DAPI (blue). Scale bar $200 \mu \mathrm{m}$. (D) Quantifications of the proportion of domeMeso hemocytes. Means and standard deviations of at least three independent experiments are presented. A total of 2612, 2350, and 4271 hemocytes were scored in control (ctr), col-/- , and RNAi col flies, respectively. ${ }^{* \star *} p<0.001$ (Student's $t$-test). (E-J) Blood cells from tub-GAL80ts, domeMeso-GAL4, UAS2x-EYFP adult flies expressing (G-J) or not (E,F) col RNAi only during adulthood. P1/NimC1 expression (red/white in lower panels) was revealed by immunostaining. Nuclei were stained with DAPI (blue). Scale bar: 20 $\mu$ m. (K) Quantifications of the proportion of domeMeso high/fusiform hemocytes (green) and domeMesolow/round hemocytes in tub-GAL80ts, domeMeso-GAL4, UAS2x-EYFP adult flies expressing (RNAi col) or not (ctr) col RNAi during adulthood. Means and standard deviations of at least three independent experiments are presented. A total of 2892 and 4489 hemocytes were scored in ctr and RNAi col flies, respectively. ${ }^{* \star} p<0.001$ (Student's $t$-test). (L-Q) Blood cells from tub-GAL80ts, domeMeso-GAL4, G-TRACE flies raised at permissive temperature (L,M) or switched from restrictive to permissive temperature $24 \mathrm{~h}$ after adult emergence (N-Q). domeMeso live expression is revealed by nuclear RFP (red) and its lineage-traced expression by nuclear GFP (green). P1/NimC1 expression (red) 


\section{FIGURE 7 | (Continued)}

was revealed by immunostaining. Nuclei were stained with DAPI. Scale bar: $20 \mu \mathrm{m}$. ctr, control non-infected flies; inf, flies infected with $E$. coli. (R) Quantifications of the proportion of domeMeso ${ }^{+}$cells expressing NimC1 in tub-GAL80ts, domeMeso-GAL4, G-TRACE flies raised at permissive temperature only during adulthood and infected (inf) or not (ctr) with E. coli. Means and standard deviations of at least seven independent experiments are presented. A total of 369 (ctr) and 449 (inf) domeMeso ${ }^{+}$cells were scored. ${ }^{* * *} p<0.001$ (Student's $t$-test). (S-Y) Representative images of the cell cycle status observed in fusiform or round hemocytes from srpD-GAL4, UAS-FUCCI control (S-U) or E. coli-infected (V-Y) adult flies. Cells in G1 phase express the nuclear GFP only, cells in G2/M express both nuclear GFP and RFP, cells in S phase express the nuclear RFP only. Cell morphology was visualized by phalloidin staining (green). Nuclei were stained with DAPI (blue). Scale bar $20 \mu \mathrm{m}$. (Z) Quantifications of the proportion of fusiform or round hemocytes in the different phase of the cell cycle in control (ctr) or in E. coli-infected (inf) srp> FUCCI adult flies. Mean values from at least six independent experiments are presented. A total of 646 (ctr) and 515 (inf) fusiform hemocytes as well as 842 (ctr) and 1336 (inf) round hemocytes were scored.

embryo/peripheral hemocytes or from the lymph gland, we performed lineage-tracing experiments with $s n$-GAL4, which is specifically expressed in embryo-derived hemocytes (AvetRochex et al., 2010), and dot-GAL4, which is specifically expressed in lymph gland-derived cells (Honti et al., 2010). In line with the idea that adult blood cells are derived from both waves of hematopoiesis (Holz et al., 2003; Sanchez Bosch et al., 2019), we observed lineage-traced expression in round hemocytes with both drivers (Figures 6M-M",P-P”). In contrast, fusiform $/ \mathrm{Col}^{+}$ cells were lineage-traced with $\operatorname{dot}-G A L 4(n=73 / 95)$ but none $(n=0 / 96)$ with $s n-G A L 4$ (Figures 6L-L",O-O" and Supplementary Figures 6C,D), demonstrating that they are solely derived from the lymph gland. Next, we asked whether fusiform cells are derived from the lymph gland posterior lobes or the PSC using, respectively, $U b x(M 3)-G A L 4$, which is expressed only in the posterior lobes (Rodrigues et al., 2021), and AntpGAL4, which is expressed in the PSC but not in the posterior lobes (Evans et al., 2009; Rodrigues et al., 2021). Importantly, we found that fusiform cells were lineage-traced with Antp-GAL4 ( $n=29 / 38$ ), while $U b x(M 3)-G A L 4$ activity was traced in round hemocytes but not in fusiform cells $(n=0 / 83)$ (Figures 6RW,R'-W',R'-W'). Furthermore, immunostaining revealed that the PSC marker Antp is specifically expressed in domeMeso ${ }^{+}$ blood cells (Supplementary Figures 6E,F). In addition, as shown for PSC cells in the larval lymph gland (Tokusumi et al., 2010), the knock-down of srp in domeMeso ${ }^{+}$cells abrogated the formation of filopodial extensions typical of these cells (Supplementary Figures 6G,H). In sum, these results show that fusiform $/ \mathrm{col}^{+} /$domeMeso ${ }^{+}$cells are derived from the PSC rather than from undifferentiated blood cells originating from the lymph gland posterior lobes.

\section{domeMeso ${ }^{+}$Cells Can Differentiate and Proliferate}

Since col is required for PSC development (Crozatier et al., 2004), we tested its role for fusiform cell development. In contrast with control flies, no domeMeso ${ }^{+}$cells (or fusiform cells) were present in $\mathrm{col}^{-/-}$adults (Figures 7A,B,D). In addition, RNAimediated knock-down of col specifically in domeMeso ${ }^{+}$cells was sufficient to ablate this lineage (Figures 7C,D). To test whether the maintenance of col expression in domeMeso ${ }^{+}$cells is required in the adult, we used a $t u b-G A L 80^{t s}$ transgene to restrict col knock-down to adulthood. Following the inhibition of col expression for $48 \mathrm{~h}$ in adult flies almost no domeMeso ${ }^{+} /$fusiform cells were retrieved, yet we observed a few cells that maintained
domeMeso expression at lower levels and adopted a round shape similar to differentiated hemocytes (Figures $7 \mathbf{E}-\mathbf{K}$ ). Interestingly, in contrast with domMeso ${ }^{\text {high }} /$ fusiform cells, these domeMeso ${ }^{\text {low }}$ cells expressed the plasmatocyte marker P1/NimC1 (Figures $7 \mathbf{H}-\mathbf{J}$ ). Similarly, in situ hybridization revealed that unlike their normal counterparts, these cells also expressed the differentiation marker $P x n$ (Supplementary Figure 7). These results show that col controls cell-autonomously domeMeso ${ }^{+}$ cells development and suggest that its expression in these cells is continuously required to prevent their differentiation.

In light of the above results, we used the G-TRACE system to test whether domeMeso ${ }^{+}$cells naturally differentiate during adulthood. Because domeMeso-GAL4 is expressed in blood cell progenitors in the larval lymph gland (Louradour et al., 2017; Rodrigues et al., 2021), we used a tubGAL80 ${ }^{\text {ts }}$ transgene to temporally restrict its activity. When domeMeso-GAL4 activity was left unrestricted (i.e., when flies were raised at $29^{\circ} \mathrm{C}$ from embryonic to adult stages), we observed lineage-traced expression of domeMeso in round cells (which expressed the plasmatocyte marker $\mathrm{P} 1 / \mathrm{NimC1}$ ) as well as live (and traced) expression in fusiform cells (Figures $7 \mathbf{L}-\mathbf{L}$ ",M-M"). When dome-GAL4 activity was restricted to the adult (i.e., when flies were raised at $18^{\circ} \mathrm{C}$ until adulthood and then switched at $29^{\circ} \mathrm{C}$ for up to 2 weeks), domeMeso $>$ G-TRACE expression was observed in fusiform cells but not in other hemocytes, except for one cell from one out of seven experiments (Figures $7 \mathbf{N}$ N",O-O",R and Supplementary Table 8). Strikingly though, we found that upon infection of adult flies by E. coli, in addition to the fusiform cells, some round/ $\mathrm{NimC1}^{+}$cells were recurrently lineage-traced with domeMeso (Figures 7P-P”,QQ",R, and Supplementary Table 8). Thus, while domeMeso ${ }^{+}$ cells do not usually differentiate during adulthood under normal conditions, they can give rise to plasmatocytes in response to an immune challenge.

Finally, it was proposed that infection of adult flies with E. coli can induce $\mathrm{Hml}^{+}$cells proliferation at low frequency (Ghosh et al., 2015), but these results were refuted by Sanchez Bosch et al. (2019), who brought several lines of evidence that $\mathrm{Hml}^{+}$ cells do not proliferate even in response to infection (Sanchez Bosch et al., 2019). Yet, $\mathrm{Hml}$ is not expressed in all blood cells and notably not in domeMeso $^{+} /$fusiform cells. We thus reassessed the cell cycle status of adult blood cells using $\operatorname{srpD-GAL4}$, which is expressed in all the hemocytes, together with the two-color FUCCI cell cycle indicator (Zielke et al., 2014). We found that $97 \%$ of the round hemocytes were in G1 phase and 3\% in G2/M but none in $S$ phase, while $73 \%$ of the fusiform cells were in G1, 
27\% in G2/M (Figures 7S-S",T-T",U-U",Z and Supplementary Table 9). Of note except for one fusiform cell in one experiment out of six, we did not observe cells in $S$ phase. These results strongly suggest that blood cell do not proliferate in uninfected adults. Following infection with $E$. coli, the proportion of round or fusiform cells in G1 or G2/M remained similar (Figures 7VV",W-W",Y-Y",Z), but in contrast with round hemocytes, a few fusiform cells (2.1\%) reproducibly entered $S$ phase (Figures 7X$\mathrm{X}$, Z and Supplementary Table 9). A similar proportion of fusiform cells (1.5\%) entered S phase upon infection of 35-dayold flies (Supplementary Table 9), suggesting that they maintain their proliferating potential during aging. In addition, when we used EdU incorporation to assess domeMeso ${ }^{+}$cell proliferation, a few domeMeso ${ }^{+}$cells $(n=8 / 370)$ incorporated EdU following infection with $E$. coli, while none were observed in control flies $(n=0 / 225)$ (Supplementary Figure 8). These results thus indicate that domeMeso $^{+} /$fusiform cells can proliferate, albeit at low frequency, in response to an immune challenge.

\section{DISCUSSION}

In mammals, the life-long production of the different blood cell types relies on the presence of long-term hematopoietic stem and progenitor cells, which are specified in the embryo and reside in specific niches from where they can be mobilized to respond to the needs of the organism (Cool and Forsberg, 2019). While several aspects of hematopoiesis are conserved from mammals to Drosophila (Banerjee et al., 2019), the presence of a similar long-term blood cell progenitor population in Drosophila remains elusive and the overall composition of the adult hematopoietic system is still a matter of debate. In particular, two successive publications came to different conclusions concerning the presence of hematopoietic progenitors and the mere existence of hematopoiesis in adult flies (Ghosh et al., 2015; Sanchez Bosch et al., 2019). Overall, our data indicate that the Drosophila adult hematopoietic system is essentially composed of different subpopulations of mature blood cells, including PSC-derived cells which seem to have some limited hematopoietic capacity, and concur with the hypothesis that no significant hematopoiesis takes place during adulthood.

Our results show that the bleeding protocol that we used ensures a swift and representative collection of the blood cell types present in the adult with minimal contamination from other tissues. This simple protocol is thus suitable to define the adult hemocyte gene repertoire without applying tissue dissociation and cell-purification protocols, which might alter gene expression. Thereby, we could show that adult hemocytes share an important part of their transcriptome with their larval parents, but also exhibit some clearly distinct features. Notably several blood cell markers are differentially expressed between adult and larval hemocytes. For instance, NimC4 and $d r p r$, which work together for apoptotic cell clearance in the embryo (Kurant et al., 2008), or Fer1HCH and Fer2LHC, which encode the two components of the iron transporter ferritin and control larval blood cell differentiation (Yoon et al., 2017), are strongly up-regulated in adult hemocytes. Moreover, our findings highlight an unexpected degree of similarities between adult and embryonic blood cells as compared to larval blood cells. Indeed, the comparison of our results with the recently described embryo versus larval blood cell gene repertoire (Cattenoz et al., 2020) revealed that almost half of the genes differentially expressed in adult versus larval hemocytes are similarly regulated in embryonic versus larval blood cells. This is particularly striking for adult up-regulated genes as 422 (out of 864) of them are also more strongly expressed in embryonic hemocytes, indicating that adult blood cells reactivate a large number of embryonic blood cell markers. Among those, many are involved in energy metabolism. Actually embryo-derived hemocytes undergo a shift from glycolysis during embryogenesis to lipid $\beta$-oxidation during the larval stages (Cattenoz et al., 2020) and our results strongly argue that a second metabolic shift occurs during adulthood. The important role of energy metabolism regulation in Drosophila blood cells is underscored by recent publications showing that fatty acid $\beta$-oxidation is required for blood cell progenitor differentiation in the larval lymph gland (Tiwari et al., 2020) and that infection leads to changes in blood cell metabolism which are important for an effective immune response (Bajgar et al., 2015; Krejcova et al., 2019). Prominent changes in energy metabolism have been observed also in mammalian blood cells in response to development, aging, infection or cancer (Rashkovan and Ferrando, 2019; Faas and de Vos, 2020; Nakamura-Ishizu et al., 2020). Getting further insights into the pathways controlling these shifts will be thus of great interest. More generally, the differences between embryonic, larval and adult blood cell gene expression programs likely reflect distinct biological functions which warrant further investigation.

Our analysis of different blood cell differentiation markers indicate that the adult hematopoietic system comprises different subtypes of plasmatocytes as well as $\sim 10 \%$ of crystal cells, but no lamellocytes. These finding are consistent with previous reports (Lanot et al., 2001; Kurucz et al., 2007; Ghosh et al., 2015; Sanchez Bosch et al., 2019), and underscore the heterogeneity of the plasmatocyte/crystal cell populations, which can be subdivided according to the expression of $\mathrm{Hml}, \mathrm{crq}$ and, to a lesser extent, $\mathrm{Cg} 25 \mathrm{C}$. It will be interesting to analyze whether these subpopulations have distinct functions for instance in the immune response or the removal of apoptotic cells, as recently shown for embryonic macrophage subpopulations (Coates et al., 2021). The proportion of these subpopulations was similar between young and aged flies, suggesting that they do not represent differentiation intermediates inherited from pupal hemocytes. Along that line, our cell ablation experiments argue against the idea that all adult hemocytes are derived from $\mathrm{Hml}^{+}$ cells or that adult $\mathrm{Hml}^{+} \mathrm{Pxn}^{+}$cells differentiate into $\mathrm{Hml}^{-} / \mathrm{Pxn}^{+}$ cells during adulthood. Besides, our observations are in line with previous reports showing that adult blood cells do not proliferate (Lanot et al., 2001; Sanchez Bosch et al., 2019) and that their number decreases with aging (Mackenzie et al., 2011; Woodcock et al., 2015; Sanchez Bosch et al., 2019). Therefore, it seems that the adult hematopoietic system is essentially composed of mature blood cells.

Ghosh et al. (2015) observed some $\mathrm{Srp}^{+}$blood cells that did not express the differentiation markers $\mathrm{NimC1}$ or Hnt and 
were lineage-traced with col-GAL4. Although the differentiation and proliferative potentials of these cells during adulthood were not assessed, the authors concluded that blood cell progenitors derived from the lymph gland posterior lobes persist during adulthood. Our results contradict this model. Indeed, while we also observed the presence of a small proportion of $s \mathrm{p}^{+}$cells that do not express hematopoietic differentiation markers and are lineage-traced as originating from the lymph gland, we demonstrated that these cells actually derive from the PSC rather than from the posterior lobes. Actually, they exhibit a characteristic fusiform shape with filopodial extensions reminiscent of PSC cells (Krzemien et al., 2007; Mandal et al., 2007), and they express Col, which is required for PSC development but also for their maintenance in the adult. Moreover, although these cells express domeMeso, they do not express other prohemocytes markers (tep4, Ance or domeGAL4), and we did not find evidence that they differentiate during adulthood in normal conditions. Nonetheless these cells hold some hematopoietic potential. Notably, upon E. coli infection, some of them can enter $S$ phase and can differentiate into plasmatocytes as evidenced by $\mathrm{NimC1}$ induction and morphological changes. Along the same line, infection of larvae with E. coli was shown to induce PSC cells proliferation and to affect their morphology (Khadilkar et al., 2017). However, given the low number of PSC-derived cells and the limited response we observed in infected adults, it is unlikely that de novo production of mature blood cells is their main function. In the lymph gland, the PSC forms a highly specialized cluster of cells that does not contribute to the general pool of blood cells but controls their fate, in particular in response to immune challenges (Crozatier et al., 2004; Benmimoun et al., 2015). It is tempting to speculate that these PSC-derived cells may have similar functions in the adult. As the knock-down of col resulted in the ablation of the PSC-derived cells and the resulting adults were viable and did not show any obvious phenotype, it would be interesting to challenge these flies with different pathogens to try to shed light on the physiological role of these cells.

In sum, our results strongly suggest that the Drosophila adult hematopoietic system does not harbor a true blood cell progenitor population with significant proliferation and differentiation potential. Still, different types of mature, potentially specialized, blood cells are present during adulthood. Along that line it will be interesting to decipher the function of the PSC-derived population, as these cells have been shown to control the cellular immune response to some specific immune challenges in the larva and may play a similar role in the adult. Finally, it is expected that applying single cell RNA-sequencing approaches to the adult hematopoietic system will bring a better description of its constituents and could reveal some of its functional features.

\section{DATA AVAILABILITY STATEMENT}

The datasets presented in this study can be found in online repositories. The names of the repository/repositories and accession number(s) can be found below: https://www.ncbi.nlm. nih.gov/geo/, GSE174107.

\section{AUTHOR CONTRIBUTIONS}

MB, FL, BB, and LW performed the experiments. YR performed bioinformatics analyses. MB, FL, YR, LV, and LW analyzed the data. MB, FL, YR, BB, LV, and LW designed the study. LW wrote the manuscript. All authors read, and approved the submitted version.

\section{FUNDING}

This work was supported by grants from the Agence Nationale de la Recherche (ANR 13-BSV3-08 and 17-CE12-0030-03), i-Site CAP20-25 and the Indo-French Centre for the Promotion of Advanced Research (IFCPAR/CEFIPRA 5503-1) to LW. MB was supported by Ph.D. fellowships from the Université ClermontAuvergne and the Fondation pour la Recherche Médicale (FRM).

\section{ACKNOWLEDGMENTS}

We thank the Toulouse RIO and Clermont-Ferrand CLIC platforms for assistance with confocal imaging. We thank all our former colleagues for valuable discussions and the Drosophila community, the Drosophila Bloomington Stock Center and the Developmental Study Hybridoma Bank (DSHB) for fly stocks and antibodies.

\section{SUPPLEMENTARY MATERIAL}

The Supplementary Material for this article can be found online at: https://www.frontiersin.org/articles/10.3389/fcell.2021. 739357/full\#supplementary-material

Supplementary Figure 1 | Venn diagrams showing the number of genes expressed (RPKM > 1 in all three biological replicates) in adult hemocytes (AD), in larval peripheral hemocytes $(\mathrm{PH})$ and in larval lymph glands (LG) as determined by RNA-seq on $w^{1118}$ females, in comparison with those annotated as hemocyte markers according to Flybase.

Supplementary Figure 2 | Confocal views showing representative bleeds from 5-day-old flies carrying the following transgenes: Cg-GAL4, UAS-RedStinger (A Cg25C>RFP), Hml $\Delta$-GAL4, UAS-RedStinger (B, Hm/ $>$ RFP), crq-RFP (C), BcF6-mCherry (D, Bc-RFP), Iz-GAL4, UAS-RedStinger (E, Iz>RFP), pxn-GAL4, $U A S-2 x E Y F P(\mathbf{F}, p x n>G F P), m s n F 9-G F P(\mathbf{G}, m s n-G F P)$, Ance-GFP (H), tep4-GAL4, UAS-2xEYFP (I, tep4> GFP), domePG125_GAL4, UAS-2xEYFP (J, dome $>$ GFP). Cells were counterstained with phalloidin (A-E: green, F-J: red) and DAPI (blue). Scale bar $200 \mu \mathrm{m}$. The percentage of cells expressing each transgene is indicated in the bottom right corner of each panel.

Supplementary Figure 3 | Confocal views of larval $(\mathbf{A}, \mathbf{B}, \mathbf{E}, \mathbf{G}, \mathbf{H}, \mathbf{K})$ and adult $\mathbf{( C , D , F , I , J , L ) ~ b l o o d ~ c e l l s ~ f r o m ~ c o n t r o l ~}(\mathbf{A}, \mathbf{C}, \mathbf{G}, \mathbf{I})$ or $\mathbf{L}$. boulardi-infected $\mathbf{( B , D , H , J )}$ $w^{1118}$ flies or from Hop ${ }^{\text {Tum }-1}$ flies $(\mathbf{E}, \mathbf{F}, \mathbf{K}, \mathbf{L})$. (A-F) Blood cells morphology was revealed with phalloidin staining (red). (G-L) Blood cells were stained with phalloidin (red) and anti- $\beta$-integrin (green). (A-L) Nuclei were stained with DAPI (blue). Scale bar $20 \mu \mathrm{m}$.

Supplementary Figure 4 | (A-H) Confocal views of hemocytes from adult flies carrying the following transgenes: srpHemo-His2A-RFP (A), Pxn-RedStinger (B), 
Cg25C-GFP (C), HmI $\triangle$-GAL4, UAS2XEYFP (D), crq-GAL4, UAS-RedStinger (E), BcF6-mCherry (F), Iz-GAL4, UAS-RedStinger (G), msnF9-mCherry (H). Cells were counterstained with phalloidin (green) and DAPI (blue). Lower panels display the red $\mathbf{( A , B , E - H )}$ or green (C,D) channel only. (I-K) Confocal views of hemocytes from domeMeso-GAL4, UAS-2XEYFP adult flies following immunostaining against P1/NimC1 (I, red) or in situ hybridization against $P x n$ (J, red) or PPO1/BC (K, red). Cell nuclei were stained with DAPI (blue). The lower panels display the red channel only. (A-K) Scale bar $20 \mu \mathrm{m}$. Left panels display round hemocytes and right panels show fusiform/domeMeso ${ }^{+}$cells.

Supplementary Figure 5 | Confocal views of abdominal hematopoietic hubs from tub-GAL80ts, Pxn-RFP, Hm/ $\Delta$-GAL4, UAS-2xEYFP control (A) and tub-GAL80ts, Pxn-RFP, HmI $\triangle$-GAL4, UAS-2xEYFP, UAS-Bax (B) flies raised at $29^{\circ} \mathrm{C}$. Cells were stained with anti-P1/NimC1 (white) and DAPI (blue). (A', B'): green (GFP) and red (RFP) channels. (A",B"): white channel (P1/NimC1). Scale bar $20 \mu \mathrm{m}$.

\section{Supplementary Figure 6 | (A-F) Confocal views of Col (A-D) or Antp (E,F)} expression in fusiform $(\mathbf{A}, \mathbf{C}, \mathbf{E})$ or round $(\mathbf{B}, \mathbf{D}, \mathbf{F})$ hemocytes from domeMeso-GAL4, UAS-2xEYFP (A,B,E,F) or dot-GAL4, G-TRACE (C,D) adult flies. (C,D) G-traced (past) activity of dot-GAL4 is visualized by nuclear GFP expression. No present expression (nuclear RFP) was observed. (G,H) Confocal views of domeMeso-GAL4, UAS-2xEYFP cells in control (G, ctr) and UAS-RNAi $\operatorname{srp}(\mathbf{H}, R N A i$ srp) adult flies. The knock-down of srp by RNAi prevents filopodia extension by domeMeso ${ }^{+}$cells. Cells were stained with DAPI (blue) and phalloidin (red). Scale bar: $20 \mu \mathrm{m}$.

Supplementary Figure 7 | Blood cells from tub-GAL80ts, domeMeso-GAL4, UAS2x-EYFP adult flies expressing (C,D) or not $(\mathbf{A}, \mathbf{B})$ col RNAi only during adulthood. Pxn expression (red) was visualized by in situ hybridization and EYFP (green) expression by immunostaining. Nuclei were stained with Topro3 (blue). The lower panels display the red channel only. Scale bar $20 \mu \mathrm{m}$. Left panels $(\mathbf{A}, \mathbf{C})$ show domeMeso ${ }^{+}$cells and right panels $(\mathbf{B}, \mathbf{D})$ show domeMesocells.

\section{REFERENCES}

Aguila, J. R., Suszko, J., Gibbs, A. G., and Hoshizaki, D. K. (2007). The role of larval fat cells in adult Drosophila melanogaster. J. Exp. Biol. 210, 956-963. doi: 10.1242/jeb.001586

Asha, H., Nagy, I., Kovacs, G., Stetson, D., Ando, I., and Dearolf, C. R. (2003). Analysis of ras-induced overproliferation in Drosophila hemocytes. Genetics 163, 203-215. doi: 10.1093/genetics/163.1.203

Avet-Rochex, A., Boyer, K., Polesello, C., Gobert, V., Osman, D., Roch, F., et al. (2010). An in vivo RNA interference screen identifies gene networks controlling Drosophila melanogaster blood cell homeostasis. BMC Dev. Biol. 10:65. doi: 10.1186/1471-213X-10-65

Ayyaz, A., Li, H., and Jasper, H. (2015). Haemocytes control stem cell activity in the Drosophila intestine. Nat. Cell. Biol. 17, 736-748. doi: 10.1038/ncb3174

Bajgar, A., Kucerova, K., Jonatova, L., Tomcala, A., Schneedorferova, I., Okrouhlik, J., et al. (2015). Extracellular adenosine mediates a systemic metabolic switch during immune response. PLoS Biol. 13:e1002135. doi: 10.1371/journal.pbio. 1002135

Banerjee, U., Girard, J. R., Goins, L. M., and Spratford, C. M. (2019). Drosophila as a genetic model for hematopoiesis. Genetics 211, 367-417. doi: 10.1534/ genetics.118.300223

Bataille, L., Auge, B., Ferjoux, G., Haenlin, M., and Waltzer, L. (2005). Resolving embryonic blood cell fate choice in Drosophila: interplay of GCM and RUNX factors. Development 132, 4635-4644. doi: 10.1242/dev.02034

Baumgardt, M., Miguel-Aliaga, I., Karlsson, D., Ekman, H., and Thor, S. (2007). Specification of neuronal identities by feedforward combinatorial coding. PLoS Biol. 5:e37. doi: 10.1371/journal.pbio.0050037

Bazzi, W., Cattenoz, P. B., Delaporte, C., Dasari, V., Sakr, R., Yuasa, Y., et al. (2018). Embryonic hematopoiesis modulates the inflammatory response and larval hematopoiesis in Drosophila. Elife 7:e34890. doi: 10.7554/eLife.34890.029

Benmimoun, B., Polesello, C., Haenlin, M., and Waltzer, L. (2015). The EBF transcription factor collier directly promotes Drosophila blood cell progenitor maintenance independently of the niche. Proc. Natl. Acad. Sci. U.S.A. 112, 9052-9057. doi: 10.1073/pnas.1423967112
Supplementary Figure 8 | (A-C) Confocal views of domeMeso-GAL4, UAS2x-EYFP blood cells from control (A) or E. coli-infected $\mathbf{( B , C )}$ adult flies fed with EdU. In vivo EdU incorporation was visualized in red. Nuclei were stained with DAPI. Scale bar $20 \mu \mathrm{m}$. The lower panels show only the green ( $\left.\mathbf{A}^{\prime}-\mathbf{C}^{\prime}\right)$ or the red (A"-C") channel.

\section{Supplementary Table 1 | List of primers used for qPCR.}

Supplementary Table $\mathbf{2}$ | List of genes expressed in adult hemocytes (RPKM > 1 in all three samples).

Supplementary Table $\mathbf{3}$ | List of genes expressed in larval peripheral hemocytes (RPKM > 1 in all three samples).

Supplementary Table $\mathbf{4}$ | List of genes expressed in larval lymph glands (RPKM > 1 in all three samples).

Supplementary Table 5 | List of hemocytes markers on Flybase (expression: hemocytes, plasmatocytes, crystal cells, lamellocytes or prohemocytes).

Supplementary Table 6 | List of differentially expressed genes between adult, larval peripheral and lymph gland blood cells (fold change $>2$, adjusted $p$-value $<0.01$.

Supplementary Table 7 | List of differentially expressed genes between embryonic and larval peripheral hemocytes (fold change $>2$, adjusted $p$-value < 0.01) (from EMBL-EBI data set: E-MTAB-8702).

Supplementary Table 8 | Quantifications of domeMeso+ cells differentiating into $\mathrm{NimC1} 1^{+}$hemocytes in control or in E. coli-infected adult flies (tubGAL80's, domeMeso > G-TRACE)

Supplementary Table $9 \mid$ Quantifications of round or fusiform hemocytes in G1, $\mathrm{S}$ or G2/M phase of the cell cycle in control or in E. coli-infected adult flies $(\operatorname{srp}>F \cup C C)$.

Benmimoun, B., Polesello, C., Waltzer, L., and Haenlin, M. (2012). Dual role for Insulin/TOR signaling in the control of hematopoietic progenitor maintenance in Drosophila. Development 139, 1713-1717. doi: 10.1242/dev.080259

Boulet, M., Miller, M., Vandel, L., and Waltzer, L. (2018). From drosophila blood cells to human leukemia. Adv. Exp. Med. Biol. 1076, 195-214. doi: 10.1007/978981-13-0529-0 11

Bourbon, H. M., Gonzy-Treboul, G., Peronnet, F., Alin, M. F., Ardourel, C., Benassayag, C., et al. (2002). A P-insertion screen identifying novel X-linked essential genes in Drosophila. Mech. Dev. 110, 71-83. doi: 10.1016/S09254773(01)00566-4

Cattenoz, P. B., Monticelli, S., Pavlidaki, A., and Giangrande, A. (2021). Toward a consensus in the repertoire of hemocytes identified in drosophila. Front. Cell Dev. Biol. 9:643712. doi: 10.3389/fcell.2021.643712

Cattenoz, P. B., Sakr, R., Pavlidaki, A., Delaporte, C., Riba, A., Molina, N., et al. (2020). Temporal specificity and heterogeneity of Drosophila immune cells. EMBO J. 39:e104486. doi: 10.15252/embj.2020104486

Charroux, B., and Royet, J. (2009). Elimination of plasmatocytes by targeted apoptosis reveals their role in multiple aspects of the Drosophila immune response. Proc. Natl. Acad. Sci. U.S.A. 106, 9797-9802. doi: 10.1073/pnas. 0903971106

Cho, B., Yoon, S. H., Lee, D., Koranteng, F., Tattikota, S. G., Cha, N., et al. (2020). Single-cell transcriptome maps of myeloid blood cell lineages in Drosophila. Nat. Commun. 11:4483. doi: 10.1101/2020.01.15.908350

Clark, R. I., Woodcock, K. J., Geissmann, F., Trouillet, C., and Dionne, M. S. (2011). Multiple TGF-beta superfamily signals modulate the adult Drosophila immune response. Curr. Biol. 21, 1672-1677. doi: 10.1016/j.cub.2011.08.048

Coates, J. A., Brooks, E., Brittle, A. L., Armitage, E. L., Zeidler, M. P., and Evans, I. R. (2021). Identification of functionally distinct macrophage subpopulations in Drosophila. Elife 10:e58686. doi: 10.7554/eLife.58686.sa2

Cool, T., and Forsberg, E. C. (2019). Chasing mavericks: the quest for defining developmental waves of hematopoiesis. Curr. Top. Dev. Biol. 132, 1-29. doi: 10.1016/bs.ctdb.2019.01.001

Crozatier, M., and Vincent, A. (1999). Requirement for the Drosophila COE transcription factor Collier in formation of an embryonic muscle: 
transcriptional response to notch signalling. Development 126, 1495-1504. doi: 10.1242/dev.126.7.1495

Crozatier, M., Ubeda, J. M., Vincent, A., and Meister, M. (2004). Cellular immune response to parasitization in Drosophila requires the EBF orthologue collier. PLoS Biol. 2:E196. doi: 10.1371/journal.pbio.0020196

de Navas, L., Foronda, D., Suzanne, M., and Sanchez-Herrero, E. (2006). A simple and efficient method to identify replacements of P-lacZ by P-Gal4 lines allows obtaining Gal4 insertions in the bithorax complex of Drosophila. Mech. Dev. 123, 860-867. doi: 10.1016/j.mod.2006.07.010

Defaye, A., Evans, I., Crozatier, M., Wood, W., Lemaitre, B., and Leulier, F. (2009). Genetic ablation of Drosophila phagocytes reveals their contribution to both development and resistance to bacterial infection. J. Innate. Immun. 1, 322-334. doi: 10.1159/000210264

Elrod-Erickson, M., Mishra, S., and Schneider, D. (2000). Interactions between the cellular and humoral immune responses in Drosophila. Curr. Biol. 10, 781-784. doi: 10.1016/S0960-9822(00)00569-8

Evans, C. J., Olson, J. M., Ngo, K. T., Kim, E., Lee, N. E., Kuoy, E., et al. (2009). G-TRACE: rapid Gal4-based cell lineage analysis in Drosophila. Nat. Methods 6, 603-605. doi: 10.1038/nmeth.1356

Faas, M. M., and de Vos, P. (2020). Mitochondrial function in immune cells in health and disease. Biochim. Biophys. Acta. Mol. Basis Dis. 1866:165845. doi: 10.1016/j.bbadis.2020.165845

Fu, Y., Huang, X., Zhang, P., van de Leemput, J., and Han, Z. (2020). Singlecell RNA sequencing identifies novel cell types in Drosophila blood. J. Genet. Genom. 47, 175-186. doi: 10.1016/j.jgg.2020.02.004

Gajewski, K. M., Sorrentino, R. P., Lee, J. H., Zhang, Q., Russell, M., and Schulz, R. A. (2007). Identification of a crystal cell-specific enhancer of the black cells prophenoloxidase gene in Drosophila. Genesis 45, 200-207. doi: 10.1002/dvg. 20285

Gaumer, S., Guenal, I., Brun, S., Theodore, L., and Mignotte, B. (2000). Bcl-2 and Bax mammalian regulators of apoptosis are functional in Drosophila. Cell Death Differ. 7, 804-814. doi: 10.1038/sj.cdd.4400714

Ghosh, S., Singh, A., Mandal, S., and Mandal, L. (2015). Active hematopoietic hubs in Drosophila adults generate hemocytes and contribute to immune response. Dev.Cell. 33, 478-488. doi: 10.1016/j.devcel.2015.03.014

Gold, K. S., and Bruckner, K. (2015). Macrophages and cellular immunity in drosophila melanogaster. Semin. Immunol. 27, 357-368. doi: 10.1016/j.smim. 2016.03.010

Grigorian, M., Mandal, L., and Hartenstein, V. (2011). Hematopoiesis at the onset of metamorphosis: terminal differentiation and dissociation of the Drosophila lymph gland. Dev. Genes. Evol. 221, 121-131. doi: 10.1007/s00427-011-0364-6

Gyoergy, A., Roblek, M., Ratheesh, A., Valoskova, K., Belyaeva, V., Wachner, S., et al. (2018). Tools allowing independent visualization and genetic manipulation of drosophila melanogaster macrophages and surrounding tissues. G3 8, 845-857. doi: 10.1534/g3.117.300452

Hartenstein, V. (2006). Blood cells and blood cell development in the animal kingdom. Annu. Rev. Cell. Dev. Biol. 22, 677-712. doi: 10.1146/annurev.cellbio. 22.010605.093317

Holz, A., Bossinger, B., Strasser, T., Janning, W., and Klapper, R. (2003). The two origins of hemocytes in Drosophila. Development 130, 4955-4962. doi: 10.1242/dev.00702

Honti, V., Csordas, G., Markus, R., Kurucz, E., Jankovics, F., and Ando, I. (2010). Cell lineage tracing reveals the plasticity of the hemocyte lineages and of the hematopoietic compartments in Drosophila melanogaster. Mol. Immunol. 47, 1997-2004. doi: 10.1016/j.molimm.2010.04.017

Irving, P., Ubeda, J. M., Doucet, D., Troxler, L., Lagueux, M., Zachary, D., et al. (2005). New insights into Drosophila larval haemocyte functions through genome-wide analysis. Cell Microbiol. 7, 335-350. doi: 10.1111/j.1462-5822. 2004.00462.x

Jung, S. H., Evans, C. J., Uemura, C., and Banerjee, U. (2005). The Drosophila lymph gland as a developmental model of hematopoiesis. Development 132, 2521-2533. doi: 10.1242/dev.01837

Khadilkar, R. J., Vogl, W., Goodwin, K., and Tanentzapf, G. (2017). Modulation of occluding junctions alters the hematopoietic niche to trigger immune activation. Elife 6:e28081. doi: 10.7554/eLife.28081.050

Kimbrell, D. A., Hice, C., Bolduc, C., Kleinhesselink, K., and Beckingham, K. (2002). The Dorothy enhancer has Tinman binding sites and drives hopscotchinduced tumor formation. Genesis 34, 23-28. doi: 10.1002/gene.10134
Krejcova, G., Danielova, A., Nedbalova, P., Kazek, M., Strych, L., Chawla, G., et al. (2019). Drosophila macrophages switch to aerobic glycolysis to mount effective antibacterial defense. Elife 8:e50414. doi: 10.7554/eLife.50414.021

Krzemien, J., Dubois, L., Makki, R., Meister, M., Vincent, A., and Crozatier, M. (2007). Control of blood cell homeostasis in Drosophila larvae by the posterior signalling centre. Nature 446, 325-328. doi: 10.1038/nature05650

Kurant, E., Axelrod, S., Leaman, D., and Gaul, U. (2008). Six-microns-under acts upstream of Draper in the glial phagocytosis of apoptotic neurons. Cell 133, 498-509. doi: 10.1016/j.cell.2008.02.052

Kurucz, E., Vaczi, B., Markus, R., Laurinyecz, B., Vilmos, P., Zsamboki, J., et al. (2007). Definition of Drosophila hemocyte subsets by cell-type specific antigens. Acta. Biol. Hung. 58, 95-111. doi: 10.1556/ABiol.58.2007.Suppl.8

Lanot, R., Zachary, D., Holder, F., and Meister, M. (2001). Postembryonic hematopoiesis in Drosophila. Dev. Biol. 230, 243-257. doi: 10.1006/dbio.2000. 0123

Lebestky, T., Chang, T., Hartenstein, V., and Banerjee, U. (2000). Specification of Drosophila hematopoietic lineage by conserved transcription factors. Science 288, 146-149. doi: 10.1126/science.288.5463.146

Leitao, A. B., and Sucena, E. (2015). Drosophila sessile hemocyte clusters are true hematopoietic tissues that regulate larval blood cell differentiation. Elife 4:e06166. doi: 10.7554/eLife.06166.021

Letourneau, M., Lapraz, F., Sharma, A., Vanzo, N., Waltzer, L., and Crozatier, M. (2016). Drosophila hematopoiesis under normal conditions and in response to immune stress. FEBS Lett. 590, 4034-4051. doi: 10.1002/1873-3468.12327

Louradour, I., Sharma, A., Morin-Poulard, I., Letourneau, M., Vincent, A., Crozatier, M., et al. (2017). Reactive oxygen species-dependent Toll/NF-kappaB activation in the Drosophila hematopoietic niche confers resistance to wasp parasitism. Elife 6:e25496. doi: 10.7554/eLife.25496.047

Luo, H., Hanratty, W. P., and Dearolf, C. R. (1995). An amino acid substitution in the Drosophila hopTum-l Jak kinase causes leukemia-like hematopoietic defects. EMBO J. 14, 1412-1420. doi: 10.1002/j.1460-2075.1995.tb07127.x

Mackenzie, D. K., Bussiere, L. F., and Tinsley, M. C. (2011). Senescence of the cellular immune response in Drosophila melanogaster. Exp. Gerontol. 46, 853-859. doi: 10.1016/j.exger.2011.07.004

Makhijani, K., Alexander, B., Tanaka, T., Rulifson, E., and Bruckner, K. (2011). The peripheral nervous system supports blood cell homing and survival in the Drosophila larva. Development 138, 5379-5391. doi: 10.1242/dev.06 7322

Mandal, L., Banerjee, U., and Hartenstein, V. (2004). Evidence for a fruit fly hemangioblast and similarities between lymph-gland hematopoiesis in fruit fly and mammal aorta-gonadal-mesonephros mesoderm. Nat. Genet. 36, 10191023. doi: $10.1038 / \mathrm{ng} 1404$

Mandal, L., Martinez-Agosto, J. A., Evans, C. J., Hartenstein, V., and Banerjee, U. A. (2007). Hedgehog- and antennapedia-dependent niche maintains Drosophila haematopoietic precursors. Nature 446, 320-324. doi: 10.1038/nature05585

Miller, M., Chen, A., Gobert, V., Auge, B., Beau, M., Burlet-Schiltz, O., et al. (2017). Control of RUNX-induced repression of Notch signaling by MLF and its partner DnaJ-1 during Drosophila hematopoiesis. PLoS Genet. 13:e1006932. doi: 10.1371/journal.pgen.1006932

Muller, H. M., Dimopoulos, G., Blass, C., and Kafatos, F. C. (1999). A hemocytelike cell line established from the malaria vector Anopheles gambiae expresses six prophenoloxidase genes. J. Biol. Chem. 274, 11727-11735. doi: 10.1074/jbc. 274.17.11727

Nakamura-Ishizu, A., Ito, K., and Suda, T. (2020). Hematopoietic stem cell metabolism during development and aging. Dev. Cell. 54, 239-255. doi: 10. 1016/j.devcel.2020.06.029

Nelliot, A., Bond, N., and Hoshizaki, D. K. (2006). Fat-body remodeling in Drosophila melanogaster. Genesis 44, 396-400. doi: 10.1002/dvg.20229

Olofsson, B., and Page, D. T. (2005). Condensation of the central nervous system in embryonic Drosophila is inhibited by blocking hemocyte migration or neural activity. Dev. Biol. 279, 233-243. doi: 10.1016/j.ydbio.2004.12.020

Oyallon, J., Vanzo, N., Krzemien, J., Morin-Poulard, I., Vincent, A., and Crozatier, M. (2016). Two independent functions of collier/early B cell factor in the control of drosophila blood cell homeostasis. PLoS One 11:e0148978. doi: 10.1371/ journal.pone.0148978

Parsons, B., and Foley, E. (2016). Cellular immune defenses of Drosophila melanogaster. Dev. Comput. Immunol. 58, 95-101. doi: 10.1016/j.dci.2015. 12.019 
Ramond, E., Dudzic, J. P., and Lemaitre, B. (2020). Comparative RNA-Seq analyses of Drosophila plasmatocytes reveal gene specific signatures in response to clean injury and septic injury. PLoS One 15:e0235294. doi: 10.1371/journal.pone. 0235294

Rashkovan, M., and Ferrando, A. (2019). Metabolic dependencies and vulnerabilities in leukemia. Genes Dev. 33, 1460-1474. doi: 10.1101/gad. 326470.119

Rodrigues, D., Renaud, Y., VijayRaghavan, K., Waltzer, L., and Inamdar, M. S. (2021). Differential activation of JAK-STAT signaling reveals functional compartmentalization in Drosophila blood progenitors. Elife 10:e61409. doi: 10.7554/eLife.61409.sa2

Sanchez Bosch, P., Makhijani, K., Herboso, L., Gold, K. S., Baginsky, R., Woodcock, K. J., et al. (2019). Adult Drosophila lack hematopoiesis but rely on a blood cell reservoir at the respiratory epithelia to relay infection signals to surrounding tissues. Dev. Cell. 51:e5. doi: 10.1016/j.devcel.2019.10.017

Sinenko, S. A., and Mathey-Prevot, B. (2004). Increased expression of Drosophila tetraspanin, Tsp68C, suppresses the abnormal proliferation of ytr-deficient and Ras/Raf-activated hemocytes. Oncogene 23, 9120-9128. doi: 10.1038/sj.onc. 1208156

Sinenko, S. A., Hung, T., Moroz, T., Tran, Q. M., Sidhu, S., Cheney, M. D., et al. (2010). Genetic manipulation of AML1-ETO-induced expansion of hematopoietic precursors in a Drosophila model. Blood 116, 4612-4620. doi: 10.1182/blood-2010-03-276998

Sorrentino, R. P., Tokusumi, T., and Schulz, R. A. (2007). The Friend of GATA protein U-shaped functions as a hematopoietic tumor suppressor in Drosophila. Dev. Biol. 311, 311-323. doi: 10.1016/j.ydbio.2007.08.011

Stramer, B., Wood, W., Galko, M. J., Redd, M. J., Jacinto, A., Parkhurst, S. M., et al. (2005). Live imaging of wound inflammation in Drosophila embryos reveals key roles for small GTPases during in vivo cell migration. J. Cell. Biol. 168, 567-573. doi: $10.1083 /$ jcb. 200405120

Tattikota, S. G., Cho, B., Liu, Y., Hu, Y., Barrera, V., Steinbaugh, M. J., et al. (2020). A single-cell survey of Drosophila blood. Elife 9:e54818. doi: 10.7554/eLife. 54818.sa2

Tepass, U., Fessler, L. I., Aziz, A., and Hartenstein, V. (1994). Embryonic origin of hemocytes and their relationship to cell death in Drosophila. Development 120, 1829-1837. doi: 10.1242/dev.120.7.1829

Tiwari, S. K., Toshniwal, A. G., Mandal, S., and Mandal, L. (2020). Fatty acid betaoxidation is required for the differentiation of larval hematopoietic progenitors in Drosophila. Elife 9:e53247. doi: 10.7554/eLife.53247.sa2

Tokusumi, T., Shoue, D. A., Tokusumi, Y., Stoller, J. R., and Schulz, R. A. (2009a). New hemocyte-specific enhancer-reporter transgenes for the analysis of hematopoiesis in Drosophila. Genesis 47, 771-774. doi: 10.1002/dvg.20561

Tokusumi, T., Sorrentino, R. P., Russell, M., Ferrarese, R., Govind, S., and Schulz, R. A. (2009b). Characterization of a lamellocyte transcriptional enhancer located within the misshapen gene of Drosophila melanogaster. PLoS One 4:e6429. doi: 10.1371/journal.pone.0006429

Tokusumi, Y., Tokusumi, T., Stoller-Conrad, J., and Schulz, R. A. (2010). Serpent, suppressor of hairless and U-shaped are crucial regulators of hedgehog niche expression and prohemocyte maintenance during Drosophila larval hematopoiesis. Development 137, 3561-3568. doi: 10.1242/dev.053728

Waltzer, L., Bataille, L., Peyrefitte, S., and Haenlin, M. (2002). Two isoforms of Serpent containing either one or two GATA zinc fingers have different roles in Drosophila haematopoiesis. EMBO J. 21, 5477-5486. doi: 10.1093/emboj/ cdf545

Woodcock, K. J., Kierdorf, K., Pouchelon, C. A., Vivancos, V., Dionne, M. S., and Geissmann, F. (2015). Macrophage-derived upd3 cytokine causes impaired glucose homeostasis and reduced lifespan in Drosophila fed a lipid-rich diet. Immunity 42, 133-144. doi: 10.1016/j.immuni.2014.12.023

Yan, Y., and Hillyer, J. F. (2020). The immune and circulatory systems are functionally integrated across insect evolution. Sci. Adv. 6:eabb3164. doi: 10. 1126/sciadv.abb3164

Yoon, S., Cho, B., Shin, M., Koranteng, F., Cha, N., and Shim, J. (2017). Iron homeostasis controls myeloid blood cell differentiation in Drosophila. Mol. Cells 40, 976-985.

Zanet, J., Jayo, A., Plaza, S., Millard, T., Parsons, M., and Stramer, B. (2012). Fascin promotes filopodia formation independent of its role in actin bundling. J. Cell. Biol. 197, 477-486. doi: 10.1083/jcb.201110135

Zielke, N., Korzelius, J., van Straaten, M., Bender, K., Schuhknecht, G. F. P., Dutta, D., et al. (2014). Fly-FUCCI: a versatile tool for studying cell proliferation in complex tissues. Cell Rep. 7, 588-598. doi: 10.1016/j.celrep.2014.03.020

Conflict of Interest: The authors declare that the research was conducted in the absence of any commercial or financial relationships that could be construed as a potential conflict of interest.

Publisher's Note: All claims expressed in this article are solely those of the authors and do not necessarily represent those of their affiliated organizations, or those of the publisher, the editors and the reviewers. Any product that may be evaluated in this article, or claim that may be made by its manufacturer, is not guaranteed or endorsed by the publisher.

Copyright (c) 2021 Boulet, Renaud, Lapraz, Benmimoun, Vandel and Waltzer. This is an open-access article distributed under the terms of the Creative Commons Attribution License (CC BY). The use, distribution or reproduction in other forums is permitted, provided the original author(s) and the copyright owner(s) are credited and that the original publication in this journal is cited, in accordance with accepted academic practice. No use, distribution or reproduction is permitted which does not comply with these terms. 